\title{
Internal Labor Markets and Worker Rents*
}

\author{
Matthias Kräkel ${ }^{\dagger}$
}

\author{
Anja Schöttner ${ }^{\ddagger}$
}

\begin{abstract}
We reconsider the concept of internal labor markets. Given homogeneous workers, we show that establishing an internal labor market by offering combined contracts across hierarchy levels strictly dominates external recruitment. This result obtains even though, in our model, firm-specific human capital is not an issue and job-promotion schemes are not necessary to provide effort incentives on lower ranks of the hierarchy. Instead, the result emerges because only an internal labor market can exploit workers' rents for incentive provision on lower tiers. Given unobservable heterogeneity of workers, relying on an internal labor market has the further advantage of improving the selection of high ability workers for higher ranks. However, observable worker heterogeneity gives rise to a trade-off between incentive and selection issues and, as a consequence, may lead to ports of entry on higher tiers of the hierarchy. Our results are in line with empirical observations that document missing elements of strict internal labor markets and traditional tournament theory in practice.
\end{abstract}

Key Words: bonuses; internal labor market; job promotion; limited liability; tournaments

JEL Classification: D82; D86; J33.

${ }^{*}$ We would like to thank Oliver Gürtler, Jenny Kragl, Patrick Schmitz, the participants of the 8th SFB/TR 15 conference on "Corporate Governance" at Chiemsee, in particular Florian Englmaier, Martin Peitz, Klaus Schmidt, Urs Schweizer, and Ernst-Ludwig von Thadden, and the participants of the 12th Colloquium on Personnel Economics in Vienna, particularly Christine Harbring, Alexander Koch, Wendelin Schnedler and Dirk Sliwka, for helpful comments. Financial support by the Deutsche Forschungsgemeinschaft (DFG), grant SFB/TR 15, is gratefully acknowledged.

${ }^{\dagger}$ University of Bonn, Adenauerallee 24-42, D-53113 Bonn, Germany, tel: +49 228 733914, fax: +49 228 739210, e-mail: m.kraekel@uni-bonn.de.

$\ddagger$ University of Bonn, Adenauerallee 24-42, D-53113 Bonn, Germany, tel: +49 228 739217, fax: +49228 739210, e-mail: anja.schoettner@uni-bonn.de. 


\section{Introduction}

Based on Doeringer and Piore (1971), Williamson, Wachter and Harris (1975), Doeringer (1986) and others, there has been a long debate on internal labor markets. In the ideal form of an internal labor market, workers enter the firm only on the lowest hierarchy level. Then, by accumulating firm-specific human capital, workers become more productive and, hence, more suitable for jobs on higher tiers of the hierarchy. Therefore, workers are promoted along structured career paths within the hierarchy. The rules of a strict internal labor market do not allow for external recruiting on higher hierarchy levels because this would diminish internal effort incentives arising from competition for promotion. Furthermore, to generate incentives via job promotion tournaments, wages should be attached to jobs on each tier of the hierarchy.

In this paper, we reconsider the theory of internal labor markets and identify new important advantages of internal promotion policies. We do so by comparing two different kinds of employment contracts. The first contract corresponds to a stylized internal labor market. We call this contractual form a combined contract because it interlinks the incentive schemes on the firm's different hierarchical tiers by a strict internal promotion rule. The second kind of contract describes just the opposite alternative as it focuses on spot contracting and allows for external hiring on higher hierarchy levels. We call this alternative separate contracts because the incentive schemes from the different hierarchical tiers do not interact: The firm chooses an optimal contract for each single tier of the hierarchy.

First, we focus on the case of homogeneous workers and show that an internal labor market in form of a combined contract dominates separate contracts. This result emerges even though we neglect the accumulation of firm-specific human capital and, moreover, in our model promotion tournaments are not essential to provide effort incentives on lower tiers of the hierarchy. In other words, two essential features of an internal labor market are missing but it still turns out to be the preferred contractual solution. The rationale for this result is as follows. We consider the realistic setting where workers are protected by limited liability and, thus, earn positive rents under any contract form. These rents lead to a specific advantage of an internal labor market in comparison to separate contracts: With an internal labor market, workers' rents can be used to enhance effort incentives for lower-level workers, which is impossible under separate contracts. As a consequence, under 
combined contracts, the firm optimally leaves larger rents to higher-level workers than under separate contracts but still incurs lower overall costs of inducing effort. Surprisingly, combined contracts may even lead to the implementation of first-best effort on higher tiers.

Recently, contract theorists as Schmitz (2005) have pointed out that optimal bonus payments that lead to positive rents can be reinterpreted as efficiency wages. Since, in general, rents are strictly increasing in effort in single-agent hidden action models with continuous effort, the implemented effort level is inefficiently small. By contrast, in our model the firm may implement first-best effort although it is associated with a strictly positive rent, which also monotonically increases in effort. Hence, in our context we obtain efficiency wages in a more literal sense.

In a second step, we introduce unobserved heterogeneity of workers so that neither the firm nor the workers themselves perfectly learn individual worker productivity. We can show that an internal labor market in form of a combined contract still dominates separate contracts. In addition to the incentive advantage under homogeneity, internal labor markets exhibit the extra benefit of improved selection quality compared to separate contracts when filling positions on higher hierarchy levels. In our setting, effort and ability of workers are complements so that both the incentive and the selection advantage reinforce each other.

Finally, our analysis points out that the strict form of an internal labor market is not always optimal: If there is observed heterogeneity of workers (i.e., the firm learns workers' abilities during the beginning of their careers), the firm can use this additional information to improve worker selection, which now may include the possibility of external recruitment on higher hierarchy levels.

The last paragraph indicates that our findings partially depart from the traditional view of strict internal labor markets. However, our results are in line with empirical facts that also contradict a strict internal labor market and, in addition, standard theory of job-promotion tournaments. ${ }^{1}$ In this sense, our paper follows the advice of Waldman (forthcoming) to develop a more sophisticated tournament model that can explain these empirical findings: We introduce a new approach that combines job-promotion tournaments with additional incentive schemes. ${ }^{2}$ Besides

\footnotetext{
${ }^{1}$ See the seminal papers by Lazear and Rosen (1981) and Nalebuff and Stiglitz (1983) on tournaments in labor economics.

${ }^{2}$ Hence, our approach builds on the general insight by Baker, Gibbs and Holmström (1994b, p. 921): "None of the major theories of wage determination can alone explain the evidence." As an alternative to our approach, Gibbons and Waldman (1999) have combined job assignment, human
} 
the fact that in organizational practice we can frequently observe the coexistence of job-promotion tournaments and bonus schemes in the same firm, our modified tournament model is in line with the following three empirical puzzles that have been highlighted particularly by Baker, Gibbs, and Holmström (1994a, 1994b): ${ }^{3}$

- First, there is considerable variation in pay on each hierarchy level, which contradicts the important prerequisite of tournaments that wages must be attached to jobs in order to generate incentives.

- Second, promotion premiums that are paid to workers when moving to higher levels in the hierarchy can explain only part of the hierarchical wage differences in firms. Baker, Gibbs, and Holmström show that often hierarchical wage differences are even five times higher (or more) than the corresponding promotion premiums.

- Third, we can observe external recruiting on higher hierarchy levels, which would erase incentives from internal job-promotion tournaments.

In our model of a two-tier hierarchy, promoted workers earn high or low bonuses depending on success or failure on the second hierarchy level. Thus, we have a natural variation in pay on the second tier, which resolves the first puzzle. As a promoted worker earns both relative performance pay and bonuses, hierarchical wage increases are only in part determined by job promotion, hence illuminating the second puzzle. In this context, one of the empirical findings by Dohmen, Kriechel, and Pfann (2004) is interesting. Contrary to other firm studies, they are able to determine the exact point in time when a worker realizes a pay increase, and they find that promotion and wage increase are often not simultaneous. This observation fits quite well to our model. Finally, under observed worker heterogeneity, the firm may hire externally with some positive probability to improve selection, which explains the third puzzle on ports of entry on higher hierarchy levels.

Our paper is related to those two tournament models that also combine a rankorder tournament with additional incentive schemes. Tsoulouhas, Knoeber, and Agrawal (2007) analyze optimal handicapping of internal and external candidates capital investments and learning in order to explain the empirical findings.

${ }^{3}$ For further empirical evidence, see Lazear (1992), Ariga, Ohkusa and Brunello (1999), Seltzer and Merrett (2000), Treble et al. (2001), Dohmen, Kriechel and Pfann (2004), Lazear and Oyer (2004), Gibbs and Hendricks (2004), and Grund (2005). 
in a contest to become CEO. To do so, they consider a promotion tournament where the prize is given by the incentive contract on the next hierarchy level. However, apart from addressing a quite different question, their model also differs from ours in several respects. First, they do not allow for relative performance pay on the first tier of the hierarchy. Second, they assume that the firm cannot commit to a secondperiod contract at the beginning of the game. ${ }^{4}$ Furthermore, even though promoted agents are of limited liability, they do not earn rents due to their high reservation utility. Schöttner and Thiele (forthcoming) also investigate incentive contracting within a two-tier hierarchy, but consider a different production environment with an individual and contractible performance signal on the first tier. They examine the optimal combination of piece rates for level-1 workers and a promotion tournament to the next tier.

Ohlendorf and Schmitz (2008) do not analyze tournaments, but combine two principal-agent contracts in successive periods. As in our model, the agent is wealthconstrained and earns a non-negative rent that can be used for incentive purposes. Compared to our paper, Ohlendorf and Schmitz consider a completely different scenario with a single agent. In their model, the principal is integrated in the production process and can invest in each of the two periods. Hence, the natural application of their model is a supplier-buyer relationship where the principal can either continue or terminate the joint project after the first period. In the OhlendorfSchmitz paper, optimal second-period incentives serve as a carrot or a stick since they depend on first-period success.

The remainder of the paper is organized as follows. In the next section, we introduce our basic model. Section 3 offers a solution to this model, comparing a combined contract with two separate contracts under homogeneous workers. In Section 4, we extend the basic model by introducing worker heterogeneity. Section 5 concludes.

\section{The Basic Model}

We consider two representative periods in the lifespan of a firm that consists of two hierarchy levels. In the first period, the firm needs to hire two workers for hierarchy level 1. In the second period, the firm has to fill one position on hierarchy level

\footnotetext{
${ }^{4}$ However, the authors also discuss an extension where commitment is possible.
} 
2. The tasks to be performed on the two hierarchy levels differ in their nature. On level 1, workers fulfill production tasks. By contrast, on level 2, we have a managerial task. Initially, we assume that all workers share the same abilities in both tasks. However, in Section 4, we also discuss the case of heterogeneous workers. Furthermore, all players are risk neutral. Workers are protected by limited liability, i.e., the firm cannot exact payments from workers. On both tiers of the hierarchy, workers have zero reservation values. For simplicity, we neglect discounting.

The tasks associated with the two hierarchical tiers lead to qualitatively different performance signals. On hierarchy level 1, a worker's effort does not generate an individually attributable output. Accordingly, the firm has only coarse information about the workers' relative contribution to firm success. Technically, it observes a non-verifiable relative performance signal that provides information about which worker has performed better. By contrast, the managerial task on level 2 is accompanied by personal responsibility and, thus, leads to an individual performance measure that is verifiable. These assumptions fit well with stylized empirical facts. Nowadays, many firms employ holistic work organization on lower hierarchy levels where the blurring of occupational barriers makes the collection of verifiable, individual performance signals prohibitively costly or even impossible. Instead, firms rely on subjective performance evaluations by workers' superiors. ${ }^{5}$ Sharper demarcation lines between jobs resulting in contractible individual performance signals are more likely to arise on managerial ranks of the hierarchy. For example, the position on level 2 may be head of a department or a division.

On the first hierarchy level, each of the two workers $i(i=A, B)$ exerts effort $\hat{e}_{i} \geq 0$. The effort has a non-verifiable monetary value $\hat{v}\left(\hat{e}_{i}\right)$ to the firm with $\hat{v}^{\prime}(\cdot)>$ 0 and $\hat{v}^{\prime \prime}(\cdot) \leq 0$. The firm neither observes $\hat{e}_{i}$ nor $\hat{v}\left(\hat{e}_{i}\right)$, but receives a non-verifiable ordinal signal $\hat{s} \in\left\{\hat{s}_{A}, \hat{s}_{B}\right\}$ about the relative performance of the two workers. The signal $\hat{s}=\hat{s}_{A}$ indicates that worker $A$ has performed best, whereas $\hat{s}=\hat{s}_{B}$ means that worker $B$ has performed better relative to his co-worker. The probability of the event $\hat{s}=\hat{s}_{A}$ is given by $\hat{p}\left(\hat{e}_{A}, \hat{e}_{B}\right)$ and that of $\hat{s}=\hat{s}_{B}$ by $1-\hat{p}\left(\hat{e}_{A}, \hat{e}_{B}\right)$.

We assume that the probability function $\hat{p}\left(\hat{e}_{A}, \hat{e}_{B}\right)$ exhibits the properties of the well-known contest-success function introduced by Dixit (1987): ${ }^{6}$

\footnotetext{
${ }^{5}$ In particular, see Ichniowski, Shaw and Prennushi (1997) and Lindbeck and Snower 2000) on holistic organizations. Subjective performance evaluation is also an integral part of the traditional personnel policy of Japanese firms; see, e.g., Koike 1988, Endo 1994.

${ }^{6}$ Subscripts of $p(\cdot, \cdot)$ denote partial derivatives.
} 
(i) $\hat{p}(\cdot, \cdot)$ is symmetric, i.e. $\hat{p}\left(\hat{e}_{i}, \hat{e}_{j}\right)=1-\hat{p}\left(\hat{e}_{j}, \hat{e}_{i}\right)$,

(ii) $\hat{p}_{1}>0, \hat{p}_{11}<0, \hat{p}_{2}<0, \hat{p}_{22}>0$,

(iii) $\hat{p}_{12}>0 \Leftrightarrow \hat{p}>0.5$.

According to (ii), exerting effort has positive but decreasing marginal returns. Property (iii) implies that if, initially, player $A$ has chosen higher effort than $B$, a marginal increase in $\hat{e}_{B}$ will make it more attractive to $A$ to increase $\hat{e}_{A}$ as well, due to the more intense competition the increase of $\hat{e}_{B}$ has caused.

Spending effort $\hat{e}_{i}$ leads to $\operatorname{costs} \hat{c}\left(\hat{e}_{i}\right)$ for worker $i(i=A, B)$ with $\hat{c}(0)=\hat{c}^{\prime}(0)=$ 0 and $\hat{c}^{\prime}\left(\hat{e}_{i}\right)>0, \hat{c}^{\prime \prime}\left(\hat{e}_{i}\right)>0$ for all $\hat{e}_{i}>0$. Furthermore, to guarantee some regularity conditions, we make the following technical assumptions. To ensure concavity of the firm's objective function, we assume that $\hat{c}^{\prime}(\hat{e}) / \hat{p}_{1}(\hat{e}, \hat{e})$ is convex for all $\hat{e}>0 .^{7}$ Finally, to obtain an interior solution, we assume that $\hat{c}^{\prime \prime}(0)=0$.

On the second hierarchy level, a worker's effort generates an individual performance signal. Following the binary-signal model by Demougin and Garvie (1991) and Demougin and Fluet (2001), we assume that the worker on level 2 chooses effort $e \geq 0$ leading to a contractible signal $s \in\left\{s^{L}, s^{H}\right\}$ on the worker's performance with $s^{H}>s^{L}$. The observation $s=s^{H}$ is favorable information about the worker's effort choice in the sense of Milgrom (1981). Let the probability of this favorable outcome be $p(e)$ with $p^{\prime}(e)>0$ (strict monotone likelihood ratio property) and $p^{\prime \prime}(e)<0$ (convexity of the distribution function condition).

If the firm assigns worker $i$ to the management task, $i$ 's effort choice $e$ yields the firm a non-verifiable monetary value $v(e)$ with $v^{\prime}(\cdot)>0$ and $v^{\prime \prime}(\cdot) \leq 0$. Again, neither $e$ nor its monetary value is observable by the firm. ${ }^{8}$ Exerting effort $e$ entails $\operatorname{costs} c(e)$ to the worker on level 2 with $c(0)=c^{\prime}(0)=0$ and $c^{\prime}(e)>0, c^{\prime \prime}(e)>0$ for all $e>0$. Furthermore, analogous to the technical assumptions for the first hierarchy level, $c^{\prime}(e) / p^{\prime}(e)$ is assumed to be convex for all $e>0,{ }^{9}$ and $c^{\prime \prime}(0)=0$.

In the given setting, the firm can use three different instruments to provide incentives: First, it can employ relative performance pay (i.e., a rank-order tournament) on hierarchy level 1. Under relative performance pay, the better performing worker receives a high wage $w_{H}$ whereas the other worker obtains a low wage $w_{L}$.

\footnotetext{
${ }^{7}$ This condition holds for a wide range of functions; for example, let effort costs be quadratic $c(\hat{e})=\hat{e}^{2}$ and the contest-success function be of the Tullock type $\hat{p}\left(\hat{e}_{A}, \hat{e}_{B}\right)=\hat{e}_{A} /\left(\hat{e}_{A}+\hat{e}_{B}\right)$.

${ }^{8}$ Note that $v(\cdot)$ measures the worker's contribution to total firm profits and is not identical with department or division profits.

${ }^{9}$ Let, for example, costs be quadratic $c(e)=e^{2}$ and the probability function be $p(e)=e^{\theta}$ with $\theta \in(0,1)$.
} 
Hence, worker $i$ earns $w_{H}$ if $\hat{s}=s_{i}$. Otherwise, he obtains $w_{L}$. Second, the firm can install a bonus scheme on hierarchy level 2. In case of a favorable signal $\left(s=s^{H}\right)$ the worker gets a high bonus $b_{H}$, whereas he receives a low bonus $b_{L}$ if the signal is bad news $\left(s=s^{L}\right)$. Due to limited liability, payments must always be non-negative $\left(w_{L}, w_{H}, b_{L}, b_{H} \geq 0\right)$. Third, the firm can interlink the two hierarchy levels by committing to establish an internal labor market (or job-promotion scheme) where the better performing worker from level 1 will be promoted to level 2 at the end of the first period. Note that, even though $\hat{s}$ is unverifiable, the firm does not have an incentive to renege on such a promotion rule because workers are homogeneous in the basic model. Consequently, it does not matter which worker is assigned to the management position. Thus, the job-promotion scheme exhibits the important self-commitment property pointed out by Malcomson $(1984,1986)$.

According to these incentive devices, the firm can offer one of the following two types of contracts. Under the first type, the firm designs separate contracts for each tier of the hierarchy. In this case, the assignment of a worker to the level-2 job is purely random: The candidate can either be hired from the external labor market or be chosen from the internal pool of workers. This kind of contract clearly differs from the ideal of an internal labor market that allows for a port of entry only on the lowest hierarchy level. By contrast, the second type of contract follows the idea of an internal labor market by combining both hierarchy levels via a job-promotion scheme (combined contract). The contract details are specified in Section 3, where we analyze incentives and worker behavior under each contractual form.

\section{Homogeneous Workers}

\subsection{Separate Contracts}

We start our analysis of the basic model with homogeneous workers by considering the case of separate contracts. Then, the time schedule of the game is as follows. First, the firm offers two workers a one-period contract specifying relative performance pay $\left(w_{L}, w_{H}\right)$ for employment on hierarchy level 1 . Provided that the workers accept the contract, they exert efforts $\hat{e}_{A}$ and $\hat{e}_{B}$, respectively. Afterwards, $\hat{s}$ is observed. The workers then obtain $w_{L}$ or $w_{H}$, respectively, whereas the firm receives $\hat{v}\left(\hat{e}_{A}\right)+\hat{v}\left(\hat{e}_{B}\right)$.

Next, the firm has to hire an individual for the management job on hierarchy 
level 2. The firm offers a randomly chosen internal or external worker a one-period contract $\left(b_{L}, b_{H}\right)$ based on the performance signal $s$ on hierarchy level 2. After acceptance of the contract, the level-2 worker chooses effort $e$ yielding either a low or a high bonus payment. The firm earns $v(e)$. The timing is summarized in the following figure.

\begin{tabular}{ccccc}
1 & 2 & 3 & 4 & 5 \\
\hline firm offers & level-1 & firm & firm offers & level-2 \\
$\left(w_{L}, w_{H}\right) ;$ & workers & observes & $\left(b_{L}, b_{H}\right) ;$ & worker \\
workers & choose $\hat{e}_{i}$ & $\hat{s} ;$ & worker & chooses $e ;$ \\
accept & & payments & accepts & payments \\
or reject & & are made & or rejects & are made
\end{tabular}

We solve the game by backwards induction and thus first analyze hierarchy level 2. For this tier, the firm's optimization problem is

$$
\begin{gathered}
\max _{b_{L}, b_{H}, e}\left\{v(e)-b_{L}-p(e) \cdot\left(b_{H}-b_{L}\right)\right\} \\
\text { s.t. } e=\arg \max _{z}\left\{b_{L}+p(z) \cdot\left(b_{H}-b_{L}\right)-c(z)\right\} \\
b_{L}+p(e) \cdot\left(b_{H}-b_{L}\right)-c(e) \geq 0 \\
b_{L}, b_{H} \geq 0 .
\end{gathered}
$$

The firm maximizes its profit net of wage payments taking into account the incentive constraint (1), the participation constraint (2), and the limited-liability constraints (3). Due to the monotone likelihood ratio property and the convexity of the distribution function condition, the incentive constraint (1) is equivalent to its first-order condition

$$
b_{H}-b_{L}=\frac{c^{\prime}(e)}{p^{\prime}(e)} .
$$


Using this relationship, the firm's problem can be transformed to

$$
\begin{gathered}
\max _{b_{L}, e}\left\{v(e)-b_{L}-p(e) \cdot \frac{c^{\prime}(e)}{p^{\prime}(e)}\right\} \\
\text { s.t. } b_{L}+p(e) \cdot \frac{c^{\prime}(e)}{p^{\prime}(e)}-c(e) \geq 0 \\
b_{L} \geq 0 .
\end{gathered}
$$

Regarding the participation constraint, we can make the following observation, which is important for our further analysis.

Lemma 1 The term

$$
r(e):=p(e) \frac{c^{\prime}(e)}{p^{\prime}(e)}-c(e)
$$

is strictly positive and monotonically increasing for all $e>0$.

Proof. $r(e)>0$ can be rewritten as $c(e)-c^{\prime}(e) \frac{p(e)}{p^{\prime}(e)}<0$. Note that $\frac{p(e)}{p^{\prime}(e)}>$ $e \Leftrightarrow p(e)-e p^{\prime}(e)>0$ is true since $p(\cdot)$ is strictly concave. But then we also have $c(e)-c^{\prime}(e) \frac{p(e)}{p^{\prime}(e)}<c(e)-e c^{\prime}(e)<0$ from the strict convexity of $c(\cdot)$. The derivative $r^{\prime}(e)=p(e)\left[\frac{c^{\prime \prime}(e) p^{\prime}(e)-p^{\prime \prime}(e) c^{\prime}(e)}{\left[p^{\prime}(e)\right]^{2}}\right]$ is positive for all $e>0$ by strict concavity of $p(e)$ and strict convexity of $c(e)$.

Hence, given $e$, the transformed participation constraint (5) is satisfied for all $b_{L} \geq 0$. Therefore, the firm optimally sets $b_{L}^{s}=0$, where superscript $s$ denotes optimal contract parameters under separate contracts. After substituting $b_{L}^{s}$ into the firm's objective function, we obtain that the firm induces the effort level $e^{s}>0$ given by ${ }^{10}$

$$
e^{s}=\arg \max _{e}\{v(e)-r(e)-c(e)\}
$$

Intuitively, since the worker is protected by limited liability, the firm has to leave him a rent. As a result, the firm's costs for inducing effort $e$ are composed of the worker's effort costs, $c(e)$, and his rent $r(e)$.

Now we turn to hierarchy level 1 . Here, two workers compete in a tournament for relative performance pay $w_{H}$ and $w_{L}$. We first characterize the workers' effort

\footnotetext{
${ }^{10}$ Due to our technical assumptions, the objective function is strictly concave; $r^{\prime \prime}(e)>0$ follows from $c^{\prime}(e) / p^{\prime}(e)$ being convex. Furthermore, the assumption $c^{\prime \prime}(0)=0$ ensures an interior solution.
} 
choices. Given the wages $w_{H}$ and $w_{L}$, worker $A$ chooses his effort level to solve

$$
\max _{\hat{e}_{A}} w_{L}+\hat{p}\left(\hat{e}_{A}, \hat{e}_{B}\right) \cdot\left[w_{H}-w_{L}\right]-\hat{c}\left(\hat{e}_{A}\right)
$$

whereas worker $B$ solves

$$
\max _{\hat{e}_{B}} w_{L}+\left[1-\hat{p}\left(\hat{e}_{A}, \hat{e}_{B}\right)\right] \cdot\left[w_{H}-w_{L}\right]-\hat{c}\left(\hat{e}_{B}\right) .
$$

The equilibrium effort levels must satisfy the first-order conditions

$$
\left(w_{H}-w_{L}\right) \hat{p}_{1}\left(\hat{e}_{A}, \hat{e}_{B}\right)=\hat{c}^{\prime}\left(\hat{e}_{A}\right) \text { and }-\left(w_{H}-w_{L}\right) \hat{p}_{2}\left(\hat{e}_{A}, \hat{e}_{B}\right)=\hat{c}^{\prime}\left(\hat{e}_{B}\right) \text {. }
$$

Recall that, due to the symmetry property (i) of the probability function $\hat{p}(\cdot, \cdot)$ we have $\hat{p}\left(\hat{e}_{B}, \hat{e}_{A}\right)=1-\hat{p}\left(\hat{e}_{A}, \hat{e}_{B}\right)$. Differentiating both sides with respect to $\hat{e}_{B}$ yields $\hat{p}_{1}\left(\hat{e}_{B}, \hat{e}_{A}\right)=-\hat{p}_{2}\left(\hat{e}_{A}, \hat{e}_{B}\right)$ so that the first-order conditions can be rewritten as

$$
w_{H}-w_{L}=\frac{\hat{c}^{\prime}\left(\hat{e}_{A}\right)}{\hat{p}_{1}\left(\hat{e}_{A}, \hat{e}_{B}\right)}=\frac{\hat{c}^{\prime}\left(\hat{e}_{B}\right)}{\hat{p}_{1}\left(\hat{e}_{B}, \hat{e}_{A}\right)} .
$$

Thus, we have a unique symmetric equilibrium $\left(\hat{e}_{A}, \hat{e}_{B}\right)=(\hat{e}, \hat{e})$ given by ${ }^{11}$

$$
w_{H}-w_{L}=\frac{\hat{c}^{\prime}(\hat{e})}{\hat{p}_{1}(\hat{e}, \hat{e})} .
$$

Condition (9) shows that equilibrium efforts increase in the tournament prize spread $w_{H}-w_{L} \cdot{ }^{12}$ To simplify notation, we denote by $\Delta w(\hat{e})$ the prize spread that induces effort $\hat{e}$, i.e.,

$$
\Delta w(\hat{e}):=\frac{\hat{c}^{\prime}(\hat{e})}{\hat{p}_{1}(\hat{e}, \hat{e})} .
$$

The firm maximizes $2 \hat{v}(\hat{e})-w_{L}-w_{H}$ subject to the incentive constraint (9),

\footnotetext{
${ }^{11}$ By strict concavity of $\hat{p}$ and convexity of $\hat{c}$, this condition is necessary and sufficient for $(\hat{e}, \hat{e})$ to be an equilibrium. Our assumptions do not rule out the existence of additional asymmetric equilibria. However, we restrict attention to the symmetric equilibrium, which is plausible in the given setting with homogeneous contestants. For example, asymmetric equilibria do not exist for the well-known Tullock or logit-form contest-success function, $\hat{p}\left(\hat{e}_{A}, \hat{e}_{B}\right)=\hat{e}_{A} /\left(\hat{e}_{A}+\hat{e}_{B}\right)$.

${ }^{12}$ We have $\frac{\partial}{\partial \hat{e}} \hat{p}_{1}(\hat{e}, \hat{e})=\hat{p}_{11}(\hat{e}, \hat{e})+\hat{p}_{12}(\hat{e}, \hat{e})<0$ due to properties (ii) and (iii) of the probability function.
} 
the participation constraint ${ }^{13}$

$$
w_{L}+\frac{1}{2}\left(w_{H}-w_{L}\right)-c(\hat{e}) \geq 0,
$$

and the limited-liability constraints

$$
w_{L}, w_{H} \geq 0
$$

Note that, when choosing the equilibrium effort $\hat{e}$, a worker must obtain at least the same expected payment as if he exerted zero effort, i.e.,

$$
w_{L}+\frac{1}{2} \Delta w(\hat{e})-c(\hat{e}) \geq w_{L}+\hat{p}(0, \hat{e}) \Delta w(\hat{e})-c(0) .
$$

Hence, $\frac{1}{2} \Delta w(\hat{e})-c(\hat{e}) \geq 0$, implying that the firm chooses $w_{L}^{s}=0$. Together with (9), it follows that $w_{H}^{s}=\Delta w(\hat{e})$ is optimal. Thus, the firm implements the effort level $\hat{e}^{s}>0$ given by ${ }^{14}$

$$
\hat{e}^{s}=\arg \max _{\hat{e}} 2 \hat{v}(\hat{e})-\Delta w(\hat{e}) .
$$

The results of this subsection are summarized in the following proposition.

Proposition 1 Under separate contracts, the firm implements the effort levels

$$
\begin{gathered}
\hat{e}^{s}=\arg \max _{\hat{e}}\{2 \hat{v}(\hat{e})-\Delta w(\hat{e})\}, \\
e^{s}=\arg \max _{e}\{v(e)-r(e)-c(e)\} .
\end{gathered}
$$

The optimal contract elements are

$$
w_{L}^{s}=0, w_{H}^{s}=\Delta w\left(\hat{e}^{s}\right), b_{L}^{s}=0, b_{H}^{s}=\frac{c^{\prime}\left(e^{s}\right)}{p^{\prime}\left(e^{s}\right)},
$$

where $r(e)$ and $\Delta w(\hat{e})$ are given by (6) and (10), respectively.

From Lemma 1, it follows that the worker on level 2 earns a strictly positive rent $r\left(e^{s}\right)$. This suggests that the firm may benefit from establishing an internal labor market where the better performing level-1 worker is promoted to the next hierarchy level. Then, the level-2 rent provides additional effort incentives for the

\footnotetext{
${ }^{13}$ In the symmetric equilibrium, each worker's winning probability is $1 / 2$.

${ }^{14}$ The second-order condition $2 \hat{v}^{\prime \prime}(\hat{e})-\Delta w^{\prime \prime}(\hat{e})<0$ is satisfied due to our technical assumption that $\hat{c}^{\prime}(\hat{e}) / \hat{p}_{1}(\hat{e}, \hat{e})$ is convex. An interior solution is guaranteed by $\hat{c}^{\prime \prime}(0)=0$.
} 
first hierarchy level. An approach that uses a strict internal promotion rule according to past performance corresponds to our combined contract, which we analyze in the following subsection.

\subsection{Combined Contract}

This subsection considers combined contracts, which correspond to a purely internal labor market. Under a combined contract, the firm offers two workers a contract $\left(w_{L}, w_{H}, b_{L}, b_{H}\right)$ at the start of the first period. The contract includes the commitment to promote the better performing level- 1 worker to level 2 in the second period, i.e., worker $i$ will be promoted if and only if $\hat{s}=\hat{s}_{i}$. Then, in the second period, the promoted worker will be paid according to the pre-specified bonus scheme. For simplicity, we assume that the worker who did not achieve promotion is dismissed. Furthermore, the worker selected for promotion can quit and realize his reservation value of zero in the second period.

The following figure summarizes the timing under a combined contract.

\begin{tabular}{ccccc}
1 & 2 & 3 & 4 & 5 \\
\hline $\begin{array}{c}\text { firm offers } \\
\left(w_{L}, w_{H},\right.\end{array}$ & level-1 & firm & firm & level-2 \\
$\left.b_{L}, b_{H}\right)$ & workers & observes & promotes & worker \\
& choose $\hat{e}_{i}$ & $\hat{s} ;$ & better & chooses $e ;$ \\
& & payments & level-1 & payments \\
& & are made & worker & are made
\end{tabular}

The time schedule differs from the one under separate contracts only with respect to stages 1 and 4 . Under a combined contract, at stage 1 , the firm offers two workers a contract $\left(w_{L}, w_{H}, b_{L}, b_{H}\right)$ that covers the following two periods. At stage 4 , the firm promotes the better level-1 worker to the next tier.

Again, the game is solved by backwards induction. In the second period, given the bonus payments $b_{L}$ and $b_{H}$, the promoted worker faces the same kind of decision problem as under separate contracts. Provided that his participation constraint (2) is satisfied, he chooses the effort level characterized by (1). In the first period, however, workers' optimization problems fundamentally differ from the case of two separate contracts. Now, increasing effort also raises the chance of being promoted 
and, consequently, earning a rent under the bonus contract. Hence, worker $A$ 's and $B$ 's optimization problems, respectively, are:

$$
\begin{aligned}
& \max _{\hat{e}_{A}} w_{L}+\hat{p}\left(\hat{e}_{A}, \hat{e}_{B}\right) \cdot\left[w_{H}-w_{L}+b_{L}+p(e)\left(b_{H}-b_{L}\right)-c(e)\right]-\hat{c}\left(\hat{e}_{A}\right) \\
& \max _{\hat{e}_{B}} w_{L}+\left[1-\hat{p}\left(\hat{e}_{A}, \hat{e}_{B}\right)\right] \cdot\left[w_{H}-w_{L}+b_{L}+p(e)\left(b_{H}-b_{L}\right)-c(e)\right]-\hat{c}\left(\hat{e}_{B}\right) .
\end{aligned}
$$

Comparing the workers' objective functions with those under separate contracts, (7) and (8), we can see that, under combined contracts, the "prize" for performing better on level 1 increases by the expected payment to the promoted worker, $b_{L}+$ $p(e)\left(b_{H}-b_{L}\right)-c(e)$. Analogously to the case of separate contracts, one can show that there is a unique symmetric equilibrium given by

$$
\hat{p}_{1}(\hat{e}, \hat{e})\left[w_{H}-w_{L}+b_{L}+p(e)\left(b_{H}-b_{L}\right)-c(e)\right]=\hat{c}^{\prime}(\hat{e})
$$

The first-period participation constraint thus is

$$
w_{L}+\frac{1}{2}\left[w_{H}-w_{L}+b_{L}+p(e)\left(b_{H}-b_{L}\right)-c(e)\right]-\hat{c}(\hat{e}) \geq 0
$$

Now we can state the firm's optimization problem: ${ }^{15}$

$$
\begin{gathered}
\max _{e, \hat{e}, w_{L}, w_{H}, b_{H}, b_{L}}\left[2 \hat{v}(\hat{e})-w_{L}-w_{H}\right]+\left[v(e)-b_{L}-p(e)\left(b_{H}-b_{L}\right)\right] \\
\text { subject to }(1),(2),(19),(20), \\
w_{L}, w_{H}, b_{L}, b_{H} \geq 0 .
\end{gathered}
$$

By solving this problem, we obtain the following result.

Proposition 2 Under a combined contract, the firm implements the effort levels

$$
\begin{gathered}
\left(\hat{e}^{c}, e^{c}\right) \in \arg \max _{\hat{e}, e}\{2 \hat{v}(\hat{e})-\Delta w(\hat{e})+v(e)-c(e)\} \\
\text { subject to } \Delta w(\hat{e})-r(e) \geq 0 .
\end{gathered}
$$

\footnotetext{
${ }^{15}$ The limited-liability constraints $b_{L}, b_{H} \geq 0$ imply that a promoted worker cannot be held liable to the extent of his tournament prize $w_{H}$. This assumption is justified when workers can use their tournament prizes for consumption before the second period ends or, alternatively, when workers are protected by a strict liability limit of zero after failure at the bonus stage. However, in the additional pages for the referees we show that replacing (23) by $w_{L} \geq 0, w_{H}+b_{L} \geq 0$ and $w_{H}+b_{H} \geq 0$ would not alter our results.
} 
The optimal contract elements are

$$
w_{L}^{c}=0, w_{H}^{c}=\Delta w\left(\hat{e}^{c}\right)-r\left(e^{c}\right), b_{L}^{c}=0, b_{H}^{c}=\frac{c^{\prime}\left(e^{c}\right)}{p^{\prime}\left(e^{c}\right)}
$$

where $r(e)$ and $\Delta w(\hat{e})$ are given by (6) and (10), respectively.

Proof. See the Appendix.

\subsection{Comparison of the Two Contracts}

Given Propositions 1 and 2, we are now able to investigate the question which of the two contracts the firm prefers to implement. Our conjecture was that the combined contract may have the advantage of partially substituting direct first-level incentives $w_{H}-w_{L}$ for indirect incentives which arise due to the prospect of the expected second-period rent $r(e)$. By comparing the optimal contract elements (16) and (26), it becomes clear that this is indeed the case because we have $w_{H}^{c}=\Delta w\left(\hat{e}^{c}\right)-r\left(e^{c}\right)$. When we examine the firm's objective functions under separate contracts (see (14) and (15)) and a combined contract (see (24)), we can see that this substitution of incentives has the consequence that the firm's costs of inducing a given pair of effort levels $(\hat{e}, e)$ are strictly lower under combined contracts: $\Delta w(\hat{e})+c(e)$ as opposed to $\Delta w(\hat{e})+r(e)+c(e)$.

However, in contrast to the case of separate contracts, the firm's optimization problem under combined contracts exhibits a constraint, (25). At first sight, one might think that this constraint restricts the set of feasible effort pairs $(\hat{e}, e)$ under combined contracts and is, thus, detrimental but such a conclusion would be wrong. As the proof of Proposition 2 shows, constraint (25) arises because, for any given level-2 effort $e$, the firm always wants to use the entire associated rent to enhance first-level incentives. In other words, given $e$, the firm wishes to implement at least the first-level effort $\hat{e}$ that workers are willing to spend to win $r(e)$, i.e., $\Delta w(\hat{e}) \geq$ $r(e)$. To induce a level-1 effort with $\Delta w(\hat{e})<r(e)$, the firm would have to punish good performance on the first tier by setting $w_{H}<w_{L}$. This cannot be optimal because the firm would actually pay for reducing effort. Hence, a combined contract only has advantages over two separate contract and is, therefore, optimal given that workers are homogeneous. ${ }^{16}$

\footnotetext{
${ }^{16}$ This is formally shown in the proof of Proposition 3.
} 
To characterize effort under the optimal combined contract, it is necessary to distinguish whether restriction (25) is binding or not at the optimum. First, assume the constraint is not binding. Then, effort on the second hierarchy level corresponds to the first-best effort level, i.e.,

$$
e^{c}=e^{F B}=\arg \max _{e}\{v(e)-c(e)\}
$$

as can be seen from (24). Hence, under the combined contract, level-2 effort is larger than under separate contracts. Concerning the first hierarchy level, however, a comparison of (14) and (24) points out that $\hat{e}^{c}=\hat{e}^{s}$. Thus, interestingly, the use of second-level rents for incentive purposes on hierarchy level 1 does not lead to higher effort on that hierarchy level. Instead, only second-tier effort increases. This result is due to the fact that raising incentives on the second tier increases efforts on both levels, but level-1 efforts are then decreased again by reducing $w_{H}-$ $w_{L}$. Hence, direct first-level incentives stemming from relative performance pay are simply replaced by indirect ones.

This observation can be related to the concept of efficiency wages, which has been reconsidered by contract theorists in the last decade. According to Tirole (1999, p. 745), Laffont and Martimort (2002, p. 174), and Schmitz (2005), efficiency wages occur if workers are protected by limited liability and earn positive rents under the optimal contract. In their models, the implemented effort level is inefficiently small. By contrast, in our setting the firm implements the efficient effort level $e^{F B}$ although this entails a strictly positive rent that is monotonically increasing in effort. Hence, combining both hierarchy levels for creating optimal incentives allows for efficiency wages in a more literal sense. As a crucial condition, the associated rent $r\left(e^{F B}\right)$ must not be too large, i.e., $\Delta w\left(\hat{e}^{s}\right)>r\left(e^{F B}\right)$. Otherwise, restriction (25) is binding at the optimal solution. In this case, $r\left(e^{F B}\right)$ exceeds the costs for inducing $\hat{e}^{s}$ under separate contracts. Implementing $e=e^{F B}$ would then yield $\hat{e}>\hat{e}^{s}$. But level-1 effort is not so valuable that the firm is willing to spend $r\left(e^{F B}\right)$. Therefore, the firm induces $e^{c}<e^{F B}$, which, however, still leads to higher level-1 effort than the optimal separate contracts.

Altogether, we have the following results:

Proposition 3 (i) The combined contract always dominates two separate contracts. (ii) If restriction (25) is non-binding, i.e., $\Delta w\left(\hat{e}^{s}\right)>r\left(e^{F B}\right)$, effort levels under the two contracts compare as follows: $\hat{e}^{c}=\hat{e}^{s}$ and $e^{c}=e^{F B}>e^{s}$. (iii) If restriction 
(25) is binding, the firm implements $\hat{e}^{c}>\hat{e}^{s}$ and $e^{F B}>e^{c}>e^{s}$.

Proof. See Appendix.

Proposition 3 shows that, when workers are homogeneous, an internal labor market with a job-promotion scheme (i.e., the combined contract) strictly dominates contractual solutions that allow external recruiting on higher hierarchy levels (i.e., separate contracts). Interestingly, strict dominance holds even though in our model internal labor markets do not foster the accumulation of firm-specific human capital and level-1 incentives can also be provided without using a job-promotion scheme, i.e., by relative performance pay.

Following the traditional view, an internal labor market has the major disadvantage of protecting workers' rents by excluding competition with external workers. In our setting, a similar situation arises: Under the combined contract, a worker's rent on hierarchy level 2 is larger than under separate contracts. However, this actually reflects a comparative advantage of internal labor markets in contrast to alternative personnel policies that allow external hiring on higher levels: By utilizing a job-promotion scheme, higher-level rents are profitably used for incentive purposes on lower hierarchical tiers. This is impossible under external recruitment. Given that an internal labor market allows the firm to use rents as an incentive device, the firm finds it optimal to leave higher rents to workers than under external recruitment.

Proposition 3 enables us to fully characterize the contracting environments that may arise in the firm with homogeneous workers. In addition to a job-promotion scheme, the firm will implement both a bonus scheme and relative performance pay if the rent for implementing first-best effort on hierarchy level 2 is not too large (case (ii) of Proposition 3). In such a situation, the firm makes use of moderate relative performance pay on the first tier by choosing a tournament winner prize $w_{H}^{c}=\Delta w\left(\hat{e}^{c}\right)-r\left(e^{F B}\right)>0$. Since $\hat{e}^{s}=\hat{e}^{c}$, the winner prize $w_{H}^{c}$ is smaller than the winner prize under separate contracts, $w_{H}^{s}=\Delta w\left(\hat{e}^{s}\right)=\Delta w\left(\hat{e}^{c}\right)$. Moreover, the firm installs high-powered incentives via a bonus system on level 2 of the hierarchy. The optimal bonus is zero in case of an unfavorable performance signal $\left(b_{L}^{c}=0\right)$. In case of a favorable signal, the worker receives the bonus $b_{H}^{c}=\frac{c^{\prime}\left(e^{F B}\right)}{p^{\prime}\left(e^{F B}\right)}$, which is larger than the high bonus under separate contracts $\left(b_{H}^{s}=\frac{c^{\prime}\left(e^{s}\right)}{p^{\prime}\left(e^{s}\right)}\right)$. However, if the rent for implementing $e^{F B}$ is rather large (case (iii) of Proposition 3), the firm foregoes 
relative performance pay on the first $\operatorname{tier}^{17}$ and, thus, solely relies on indirect level-1 incentives through the second-period rent.

Our results are nicely in line with two empirical observations that contradict standard models on job-promotion tournaments. Baker, Gibbs, and Holmström (1994a, 1994b) analyze the internal structure and the wage policy of a US corporation. ${ }^{18}$ As a first puzzling result, they find considerable variation in pay on each hierarchy level (see Figure VI in Baker, Gibbs and, Holmström 1994a, p. 906). This finding contradicts the important prerequisite of standard job-promotion tournaments that wages must be attached to jobs and, therefore, to hierarchy levels in order to generate incentives. In our model, under the combined contract we have a job-promotion tournament with pay variation because the promoted worker may or may not receive a bonus on hierarchy level 2 .

Furthermore, according to standard job-promotion tournaments, hierarchical wage differences should be completely explained by promotion premiums paid to workers when moving to higher levels in the hierarchy. However, Baker, Gibbs, and Holmström (1994a) find that "promotion premiums explain only part of the differences in pay between levels" (p. 909). In fact, often hierarchical wage differences are even five times higher (or more) than the corresponding promotion premiums. This second puzzling observation is also in line with our modified tournament model. Under the combined contract, a promoted worker does not only earn the promotion premium $w_{H}-w_{L}$ but may also receive a bonus. In particular, the higher the expected rent on hierarchy level 2, the smaller will be the optimal promotion premium. The reason is that indirect incentives replace direct ones. Presuming that effort on higher hierarchy levels is more valuable to firms than effort choices on lower levels, ${ }^{19}$ we will have considerable rents on higher tiers, thus reducing corresponding promotion premiums.

So far, we have shown that an internal labor market structure in form of a combined contract with a job-promotion scheme is optimal when workers are ho-

\footnotetext{
${ }^{17}$ Note that, since $\Delta w\left(\hat{e}^{c}\right)=r\left(e^{c}\right)$, we must have $w_{H}^{c}=0$.

${ }^{18}$ The empirical puzzles documented by Baker, Gibbs, and Holmström (1994a, 1994b) are also found by Treble et al. (2001), who analyzed a British firm. Considerable wage variation within job levels is also documented by the empirical studies of Seltzer and Merrett (2000), Dohmen, Kriechel and Pfann (2004), Gibbs and Hendricks (2004) and Grund (2005). Moreover, Dohmen, Kriechel and Pfann (2004) show that promotion and wage increase are often not simultaneous, which gives further evidence that salaries are also determined by bonuses and not solely by promotion premiums.

${ }^{19}$ That is, the firm's value function for effort increases more steeply on higher hierarchy levels.
} 
mogeneous. However, in practice workers typically differ in their abilities. In that case, selection of high-ability workers becomes an issue. In the next section, we therefore consider worker heterogeneity and investigate whether an internal labor market is still optimal.

\section{Heterogeneous Workers}

In this section, we extend our basic model to two situations where workers potentially differ in their abilities. In one situation, we assume that a worker's ability is revealed to the firm after one period of interaction. Such characteristics could be soft skills such as social competence, the capability to lead and motivate people, or to oversee complex production processes. However, other characteristics of a worker's ability may remain unrevealed for some time, which is the other situation that we analyze. We start with the latter case of unobserved heterogeneity, where workers' abilities do not become observable during the two periods of employment. In Subsection 4.2, we analyze a situation in which the firm learns individual worker abilities during the first period. The firm can use this information for filling the management position on the second tier. Thus, in both situations, the incentive problem is now supplemented by a selection problem.

\subsection{Unobserved Worker Heterogeneity}

\subsubsection{Modifications of the Basic Model}

Neither the firm nor the workers themselves can observe worker abilities during the two periods of employment. We assume that this aspect of unobserved ability persists over time and hierarchy levels, i.e., it is not task specific. Because it is not possible to observe a worker's individual output on the first hierarchy level, the firm cannot deduce marginal productivity at the end of the first period. Let each worker either have high unobservable talent $t_{1}$ or low unobservable talent $t_{0}$ with $t_{1}>t_{0}>0$. Neither the workers nor the firm observe the workers' individual talents during the whole game. In other words, we introduce symmetric uncertainty about the quality of the workers. ${ }^{20}$ All players (i.e., the workers and the firm) have the

\footnotetext{
${ }^{20}$ The assumption of symmetric talent uncertainty is widespread in labor economics. See, among many others, Harris and Holmström (1982), Murphy (1986), Holmström (1999) and Gibbons and Waldman (1999).
} 
same prior distribution about worker talent. For simplicity, let each talent be equally likely so that unobservable talent can be described by a random variable $t$ that takes values $t_{0}$ and $t_{1}$ with probability $\frac{1}{2}$, respectively, and has mean $E[t]=\left(t_{0}+t_{1}\right) / 2$.

On each hierarchy level, a worker's talent influences both the value of effort for the firm and the probability of generating a favorable signal. Let the value of worker $i(i=A, B)$ to the firm when exerting effort $\hat{e}_{i}$ on level 1 be $t \cdot \hat{v}\left(\hat{e}_{i}\right)$, and that on level 2 when choosing effort $e$ be $t \cdot v(e)$. In analogy, the probability of a favorable signal on level 2 is now given by $t \cdot p(e)$, with $t_{1} \cdot p(e) \leq 1$ for all $e$. For a relative performance signal on level 1 we have to differentiate four possible situations. If both workers have equal talents, $A$ 's probability of winning the tournament will again be described by the function $\hat{p}\left(\hat{e}_{A}, \hat{e}_{B}\right)$. In addition, now we also have two possible asymmetric pairings. If worker $A$ has high talent $t_{1}$ and worker $B$ low talent $t_{0}$, A's probability of getting the better evaluation will be described by $\hat{p}\left(\hat{e}_{A}, \hat{e}_{B} ; t_{1}\right)$ whereas $B$ 's one is given by $1-\hat{p}\left(\hat{e}_{A}, \hat{e}_{B} ; t_{1}\right)$. In the opposite asymmetric case with $B$ being more talented than $A$, worker $A$ wins the tournament with probability $\hat{p}\left(\hat{e}_{A}, \hat{e}_{B} ; t_{0}\right)$ and $B$ with probability $1-\hat{p}\left(\hat{e}_{A}, \hat{e}_{B} ; t_{0}\right)$.

We assume that the new probability functions have analogous properties (i)(iii) as the function $\hat{p}(\cdot, \cdot)$ (see Section 2). For example, in the basic model we have $\hat{p}_{1}\left(\hat{e}_{j}, \hat{e}_{i}\right)=-\hat{p}_{2}\left(\hat{e}_{i}, \hat{e}_{j}\right)$, which follows from the symmetry assumption (i). In analogy, we assume that also in heterogeneous pairings the specific identity of a certain worker does not have any influence on his (marginal) winning probability, that is whether a worker acts on the first or on the second position in $\hat{p}(\cdot, \cdot ; t)$ does not influence the (marginal) returns of his effort choice for a given asymmetric pairing. Technically, this means that $\hat{p}\left(\hat{e}_{i}, \hat{e}_{j} ; t_{1}\right)=1-\hat{p}\left(\hat{e}_{j}, \hat{e}_{i} ; t_{0}\right)$, implying

$$
\hat{p}_{1}\left(\hat{e}_{i}, \hat{e}_{j} ; t_{1}\right)=-\hat{p}_{2}\left(\hat{e}_{j}, \hat{e}_{i} ; t_{0}\right) \text { and } \hat{p}_{2}\left(\hat{e}_{i}, \hat{e}_{j} ; t_{1}\right)=-\hat{p}_{1}\left(\hat{e}_{j}, \hat{e}_{i} ; t_{0}\right)
$$

for $i, j=A, B ; i \neq j$. Of course, talent should have an impact on a worker's absolute winning probability and his marginal one. In particular, we assume that, for given effort levels, the more talented worker has a higher winning probability than the less talented one, i.e.,

$$
\hat{p}\left(\hat{e}_{i}, \hat{e}_{j} ; t_{1}\right)>\hat{p}\left(\hat{e}_{i}, \hat{e}_{j} ; t_{0}\right) .
$$


Furthermore, let effort and talent be complements in the sense of

$$
\hat{p}_{1}\left(\hat{e}_{i}, \hat{e}_{j} ; t_{1}\right)>\hat{p}_{1}\left(\hat{e}_{i}, \hat{e}_{j} ; t_{0}\right) \text { and }-\hat{p}_{2}\left(\hat{e}_{i}, \hat{e}_{j} ; t_{0}\right)>-\hat{p}_{2}\left(\hat{e}_{i}, \hat{e}_{j} ; t_{1}\right)
$$

that is marginally increasing effort is more effective under high talent than under low one. Properties (ii) and (iii) from the basic model also hold analogously for heterogeneous workers. Note that property (iii) together with symmetry here implies that $\hat{p}_{12}\left(\hat{e}, \hat{e} ; t_{1}\right)=-\hat{p}_{12}\left(\hat{e}, \hat{e} ; t_{0}\right)$ : If workers choose identical efforts the more able one has a higher winning probability; if now the other worker increases his effort, competition becomes more intense so that the more able worker raises his effort, too. Again, this effect is assumed to be independent of whether a worker acts on the first or on the second position in $\hat{p}(\cdot, \cdot ; t)$. Finally, we assume analogous regularity conditions to hold as in the basic model of Section 2.

In the following, we will investigate how the comparison between separate contracts and a combined contract will change when workers are characterized by unobserved heterogeneity.

\subsubsection{Separate Contracts}

We first consider the case of separate contracts. The equilibrium on hierarchy level 1 is now described by the first-order conditions

$$
\begin{gathered}
\left(w_{H}-w_{L}\right) \frac{1}{4}\left(\hat{p}_{1}\left(\hat{e}_{A}, \hat{e}_{B} ; t_{1}\right)+\hat{p}_{1}\left(\hat{e}_{A}, \hat{e}_{B} ; t_{0}\right)+2 \hat{p}_{1}\left(\hat{e}_{A}, \hat{e}_{B}\right)\right)=\hat{c}^{\prime}\left(\hat{e}_{A}\right), \\
\left(w_{H}-w_{L}\right) \frac{1}{4}\left(-\hat{p}_{2}\left(\hat{e}_{A}, \hat{e}_{B} ; t_{1}\right)-\hat{p}_{2}\left(\hat{e}_{A}, \hat{e}_{B} ; t_{0}\right)-2 \hat{p}_{2}\left(\hat{e}_{A}, \hat{e}_{B}\right)\right)=\hat{c}^{\prime}\left(\hat{e}_{B}\right) .
\end{gathered}
$$

Using $\hat{p}_{1}\left(\hat{e}_{B}, \hat{e}_{A}\right)=-\hat{p}_{2}\left(\hat{e}_{A}, \hat{e}_{B}\right)$ and $(27)$ shows that there exists a symmetric equilibrium in which each worker chooses $\hat{e}$ characterized by

$$
\begin{gathered}
w_{H}-w_{L}=\Delta \tilde{w}(\hat{e}) \\
\text { with } \Delta \tilde{w}(\hat{e}):=\frac{4 \hat{c}^{\prime}(\hat{e})}{\hat{p}_{1}\left(\hat{e}, \hat{e} ; t_{1}\right)+\hat{p}_{1}\left(\hat{e}, \hat{e} ; t_{0}\right)+2 \hat{p}_{1}(\hat{e}, \hat{e})}
\end{gathered}
$$


and $\Delta \tilde{w}^{\prime}(\hat{e})>0 .{ }^{21}$ The firm maximizes $2 E[t] \hat{v}(\hat{e})-w_{L}-w_{H}$ subject to the participation constraint (11), ${ }^{22}$ the limited-liability constraints (12) and the incentive constraint (30). The optimal tournament prizes are, therefore, given by $w_{L}^{s}=0$ and $w_{H}^{s}=\Delta \tilde{w}(\hat{e})$, and the firm implements the effort level ${ }^{23} \hat{e}_{h}^{s}$ that solves

$$
\max _{\hat{e}} 2 E[t] \hat{v}(\hat{e})-\Delta \tilde{w}(\hat{e})
$$

On hierarchy level 2, the firm's optimization problem now reads as

$$
\begin{gathered}
\max _{b_{L}, b_{H}, e}\left\{E[t] v(e)-b_{L}-E[t] p(e)\left(b_{H}-b_{L}\right)\right\} \\
\text { subject to } e=\arg \max _{z}\left\{b_{L}+E[t] p(z)\left(b_{H}-b_{L}\right)-c(z)\right\} \\
b_{L}+E[t] p(e)\left(b_{H}-b_{L}\right)-c(e) \geq 0 \\
b_{L}, b_{H} \geq 0 .
\end{gathered}
$$

In analogy to the basic model, the incentive constraint can be replaced with the first-order condition $b_{H}-b_{L}=\frac{c^{\prime}(e)}{E[t] p^{\prime}(e)}$. It is straightforward to show that, under the optimal bonus contract, we have $b_{L}^{s}=0$. Furthermore, the participation constraint is identical to (5) and the firm thus implements effort $e_{h}^{s}$ with

$$
e_{h}^{s}=\arg \max _{e}\{E[t] v(e)-r(e)-c(e)\}
$$

and $r(e)$ being defined in (6). Altogether, the comparison of (32) and (33) with (14) and (15) from the basic model shows that introducing unobserved heterogeneity leads to changes in the expected values of the workers' effort choices and in the optimal winner prize $w_{H}^{*}$, but leaves the implementation costs on level 2 unchanged for a given effort level $e$.

\subsubsection{Combined Contract}

Now we turn to the analysis of the combined contract. Solving the game by backwards induction, we first consider the actions on hierarchy level 2. Here, all players

\footnotetext{
${ }^{21}$ Note that $\frac{\partial}{\partial \hat{e}}\left(\hat{p}_{1}\left(\hat{e}, \hat{e} ; t_{1}\right)+\hat{p}_{1}\left(\hat{e}, \hat{e} ; t_{0}\right)+2 \hat{p}_{1}(\hat{e}, \hat{e})\right)=\hat{p}_{11}\left(\hat{e}, \hat{e} ; t_{1}\right)+\hat{p}_{12}\left(\hat{e}, \hat{e} ; t_{1}\right)+\hat{p}_{11}\left(\hat{e}, \hat{e} ; t_{0}\right)$ $+\hat{p}_{12}\left(\hat{e}, \hat{e} ; t_{0}\right)+2 \hat{p}_{11}(\hat{e}, \hat{e})+2 \hat{p}_{12}(\hat{e}, \hat{e})<0$.

${ }^{22}$ Note that, due to the symmetric equilibrium, the participation constraint will be the same as in the basic model.

${ }^{23}$ Here and in the following, the subscript " $h$ " for optimal efforts indicates heterogeneity of workers.
} 
update their beliefs about the unknown talent of the promoted worker. Let $E[t \mid \hat{s}]$ denote the expected talent of the promoted worker, that is each player calculates a new expectation depending on the realization of the relative performance signal $\hat{s}$. Note that at any prior point in time the workers as well as the firm already know that they have to update their beliefs in light of the promotion decision and that they will not receive further information. Hence, when designing the optimal combined contract, the firm has to include the incentive constraint

$$
b_{H}-b_{L}=\frac{c^{\prime}(e)}{E[t \mid \hat{s}] p^{\prime}(e)}
$$

and the participation constraint

$$
b_{L}+E[t \mid \hat{s}] p(e)\left(b_{H}-b_{L}\right)-c(e) \geq 0 \Leftrightarrow b_{L}+r(e) \geq 0
$$

where the last inequality follows from (6) and (34).

At level 1, worker $A$ and worker $B$ maximize

$$
\begin{aligned}
& w_{L}+\left(w_{H}-w_{L}+b_{L}+E[t \mid \hat{s}] p(e)\left(b_{H}-b_{L}\right)-c(e)\right) \\
& \times \frac{1}{4}\left(\hat{p}\left(\hat{e}_{A}, \hat{e}_{B} ; t_{1}\right)+\hat{p}\left(\hat{e}_{A}, \hat{e}_{B} ; t_{0}\right)+2 \hat{p}\left(\hat{e}_{A}, \hat{e}_{B}\right)\right)-\hat{c}\left(\hat{e}_{A}\right) \text { and } \\
& w_{L}+\left(w_{H}-w_{L}+b_{L}+E[t \mid \hat{s}] p(e)\left(b_{H}-b_{L}\right)-c(e)\right) \\
& \times \frac{1}{4}\left(\left(1-\hat{p}\left(\hat{e}_{A}, \hat{e}_{B} ; t_{1}\right)\right)+\left(1-\hat{p}\left(\hat{e}_{A}, \hat{e}_{B} ; t_{0}\right)\right)+2\left(1-\hat{p}\left(\hat{e}_{A}, \hat{e}_{B}\right)\right)\right)-\hat{c}\left(\hat{e}_{B}\right),
\end{aligned}
$$

respectively. Equations (6) and (34) together with the first-order conditions, $\hat{p}_{1}\left(\hat{e}_{B}, \hat{e}_{A}\right)=$ $-\hat{p}_{2}\left(\hat{e}_{A}, \hat{e}_{B}\right)$ and $(27)$ yield

$$
\begin{gathered}
\left(w_{H}-w_{L}+b_{L}+r(e)\right) \frac{\hat{p}_{1}\left(\hat{e}_{A}, \hat{e}_{B} ; t_{1}\right)+\hat{p}_{1}\left(\hat{e}_{A}, \hat{e}_{B} ; t_{0}\right)+2 \hat{p}_{1}\left(\hat{e}_{A}, \hat{e}_{B}\right)}{4}=\hat{c}^{\prime}\left(\hat{e}_{A}\right) \\
\left(w_{H}-w_{L}+b_{L}+r(e)\right) \frac{\hat{p}_{1}\left(\hat{e}_{B}, \hat{e}_{A} ; t_{0}\right)+\hat{p}_{1}\left(\hat{e}_{B}, \hat{e}_{A} ; t_{1}\right)+2 \hat{p}\left(\hat{e}_{B}, \hat{e}_{A}\right)}{4}=\hat{c}^{\prime}\left(\hat{e}_{B}\right) .
\end{gathered}
$$

Thus, in the symmetric equilibrium each worker exerts $\hat{e}$ described by

$$
w_{H}-w_{L}+b_{L}+r(e)=\Delta \tilde{w}(\hat{e})
$$

with $\Delta \tilde{w}(\hat{e})$ being defined in (31). 
Now we can state the firm's problem. It maximizes

$$
\begin{gathered}
2 E[t] \hat{v}(\hat{e})-2 w_{L}-\left(w_{H}-w_{L}\right)+E[t \mid \hat{s}] v(e)-b_{L}-E[t \mid \hat{s}] p(e)\left(b_{H}-b_{L}\right) \\
\stackrel{(6),(34),(36)}{=} 2 E[t] \hat{v}(\hat{e})-\Delta \tilde{w}(\hat{e})+E[t \mid \hat{s}] v(e)-2 w_{L}-c(e)
\end{gathered}
$$

subject to the limited-liability constraints (23), the incentive constraints (34) and (36), the participation constraint for the second hierarchy level (35) and the participation constraint for the first level,

$$
\begin{array}{r}
w_{L}+\frac{1}{2}\left(w_{H}-w_{L}+b_{L}+E[t \mid \hat{s}] p(e)\left(b_{H}-b_{L}\right)-c(e)\right)-\hat{c}(\hat{e}) \geq 0 \\
\stackrel{(6),(34),(36)}{\Leftrightarrow} w_{L}+\frac{1}{2} \Delta \tilde{w}(\hat{e})-\hat{c}(\hat{e}) \geq 0 .
\end{array}
$$

Moreover, the firm has to take into account that $E[t \mid \hat{s}]$ depends on the workers' equilibrium efforts chosen on hierarchy level 1:

$$
\begin{aligned}
E[t \mid \hat{s}] & =\frac{1}{4} t_{1}+\frac{1}{4} t_{0}+\frac{1}{4}\left(\hat{p}\left(\hat{e}, \hat{e} ; t_{1}\right) t_{1}+\left(1-\hat{p}\left(\hat{e}, \hat{e} ; t_{1}\right)\right) t_{0}\right) \\
& +\frac{1}{4}\left(\hat{p}\left(\hat{e}, \hat{e} ; t_{0}\right) t_{0}+\left(1-\hat{p}\left(\hat{e}, \hat{e} ; t_{0}\right)\right) t_{1}\right) \\
& =E[t]+\frac{\Delta t\left(\hat{p}\left(\hat{e}, \hat{e} ; t_{1}\right)-\hat{p}\left(\hat{e}, \hat{e} ; t_{0}\right)\right)}{4} \stackrel{(28)}{>} E[t]
\end{aligned}
$$

with $\Delta t:=t_{1}-t_{0}$. Thus, the posterior expectation is larger than the prior one because the more talented worker is promoted with higher probability in case of an asymmetric pairing in the tournament. Furthermore, the posterior mean strictly increases in level-1 equilibrium efforts as talent and effort are complements:

$$
\begin{aligned}
\frac{\partial E[t \mid \hat{s}]}{\partial \hat{e}} & =\frac{\Delta t}{4}\left(\hat{p}_{1}\left(\hat{e}, \hat{e} ; t_{1}\right)+\hat{p}_{2}\left(\hat{e}, \hat{e} ; t_{1}\right)-\hat{p}_{1}\left(\hat{e}, \hat{e} ; t_{0}\right)-\hat{p}_{2}\left(\hat{e}, \hat{e} ; t_{0}\right)\right) \\
& \stackrel{(27)}{=} \frac{\Delta t}{2}\left(\hat{p}_{1}\left(\hat{e}, \hat{e} ; t_{1}\right)-\hat{p}_{1}\left(\hat{e}, \hat{e} ; t_{0}\right)\right) \stackrel{(29)}{>} 0
\end{aligned}
$$

Applying the same two-step procedure as in the basic model yields that the firm 
implements the effort pair $\left(\hat{e}_{h}^{c}, e_{h}^{c}\right)$ with $^{24}$

$$
\begin{gathered}
\left(\hat{e}_{h}^{c}, e_{h}^{c}\right) \in \arg \max _{\hat{e}, e}\{2 E[t] \hat{v}(\hat{e})+E[t \mid \hat{s}] v(e)-\Delta \tilde{w}(\hat{e})-c(e)\} \\
\text { subject to } \Delta \tilde{w}(\hat{e})-r(e) \geq 0 .
\end{gathered}
$$

When comparing optimal efforts under the combined contract with those under two separate contracts, we have to distinguish whether the restriction (40) is binding or not at the optimum. In case of a non-binding restriction, optimal efforts $\left(\hat{e}_{h}^{c}, e_{h}^{c}\right)$ are described by the first-order conditions

$$
2 E[t] \hat{v}^{\prime}(\hat{e})+\frac{\partial E[t \mid \hat{s}]}{\partial \hat{e}} v(e)=\Delta \tilde{w}^{\prime}(\hat{e}) \quad \text { and } \quad E[t \mid \hat{s}] v^{\prime}(e)=c^{\prime}(e) .
$$

Comparing the first equation with (32) clearly shows that $\hat{e}_{h}^{c}>\hat{e}_{h}^{s}$ as $\partial E[t \mid \hat{s}] /$ $\partial \hat{e}>0$. The comparison of the second equation with (33) points out that $e_{h}^{c}>e_{h}^{s}$, due to Lemma 1 and the fact that $E[t \mid \hat{s}]>E[t]$. Now, we have to consider the case of a binding restriction (40). Using this restriction, we can express level-2 effort as a function of level-1 effort, $e(\hat{e})$, with $\frac{\partial e}{\partial \hat{e}}=\frac{\Delta \tilde{w}^{\prime}(\hat{e})}{r^{\prime}(e)}>0$. The firm's objective function under a combined contract can be rewritten as

$$
2 E[t] \hat{v}(\hat{e})+E[t \mid \hat{s}] v(e(\hat{e}))-\Delta \tilde{w}(\hat{e})-c(e(\hat{e}))
$$

The first-order condition yields

$$
2 E[t] \hat{v}^{\prime}(\hat{e})+\frac{\partial E[t \mid \hat{s}]}{\partial \hat{e}} v(e(\hat{e}))-\Delta \tilde{w}^{\prime}(\hat{e})+\left[E[t \mid \hat{s}] v^{\prime}(e(\hat{e}))-c^{\prime}(e(\hat{e}))\right] \frac{\partial e}{\partial \hat{e}}=0 .
$$

Inserting for $\partial e / \partial \hat{e}$ leads to

$$
2 E[t] \hat{v}^{\prime}(\hat{e})+\frac{\partial E[t \mid \hat{s}]}{\partial \hat{e}} v(e(\hat{e}))+\frac{E[t \mid \hat{s}] v^{\prime}(e(\hat{e}))-c^{\prime}(e(\hat{e}))-r^{\prime}(e(\hat{e}))}{r^{\prime}(e(\hat{e}))} \Delta \tilde{w}^{\prime}(\hat{e})=0
$$

Since the first two expressions as well as $r^{\prime}(e(\hat{e}))$ and $\Delta \tilde{w}^{\prime}(\hat{e})$ are positive, the numerator of the last expression is negative. As this numerator is a strictly concave function of $e(\hat{e})$ and since $E[t \mid \hat{s}]>E[t]$, we obtain from the comparison with (33) that $e_{h}^{c}>e_{h}^{s}$.

Finally, we have to consider optimal effort implementation on hierarchy level 1.

\footnotetext{
${ }^{24}$ See the additional pages for the referees.
} 
Since (40) is binding, the effort $\hat{e}$ that would maximize level 1-profit corresponds to a level-2 effort that is below the effort $e$ that maximizes level-2 profit $E[t \mid \hat{s}] v(e)-$ $c(e)$. Hence, the firm may be interested in further raising $\hat{e}$. As both profit functions are strictly concave, we can apply the same argument as in the proof of Proposition 3: The firm would, thus, never implement a smaller $\hat{e}$ than the optimal effort under a non-binding restriction. Since that effort was larger than the optimal level-1 effort under separate contracts, we have proved that $\hat{e}_{h}^{c}>\hat{e}_{h}^{s}$ also holds under a binding restriction.

Proposition 4 Irrespective of whether restriction (40) is binding or not at the optimum, we have $\hat{e}_{h}^{c}>\hat{e}_{h}^{s}$ and $e_{h}^{c}>e_{h}^{s}$.

Proposition 4 points out that, under a combined contract, the firm implements strictly larger efforts on hierarchy level 1 than under separate contracts. This result sharply contrasts with our findings in Proposition 3 on homogeneous workers. The intuition comes from the fact that, in case of unobservable talent, the firm has an additional motive for implementing large efforts on hierarchy level 1: The larger $\hat{e}$ the higher will be the probability that the worker of higher unobserved talent is promoted to level 2 in case of a heterogeneous pairing, i.e., $\hat{p}_{1}\left(\hat{e}, \hat{e} ; t_{1}\right)>0$. This, in turn, increases the posterior expected talent of the promoted worker: $\partial E[t \mid \hat{s}] / \partial \hat{e}>$ 0 according to $(38)$ since $E[t \mid \hat{s}]$ monotonically increases in $\hat{p}\left(\hat{e}, \hat{e} ; t_{1}\right)$. In other words, if workers have unobservable characteristics that persist across hierarchy levels, higher incentives on level 1 improve worker selection for level 2. The reason is that incentives and selection are strictly interlinked.

Again, from a pure incentive perspective, the firm is strictly better off by choosing a combined contract. Analogously to the basic model, the combined contract will lead to first-best effort on hierarchy level 2, i.e. $e_{h}^{c}=\arg \max _{e}\{E[t \mid \hat{s}] v(e)-c(e)\}$, if restriction (40) is not binding. However, there is a crucial difference in comparison to the basic model. With unobserved talents, we have the additional effect that combining both hierarchy levels via a job-promotion scheme even improves on first-best implementation under uncertainty as $E[t \mid \hat{s}]>E[t]$. By inducing large efforts $\hat{e}$ on level 1, the firm raises the posterior expected talent of the promoted worker (i.e. $\partial E[t \mid \hat{s}] / \partial \hat{e}>0$ ) which, in turn, increases the efficient effort level $e_{h}^{c}$ on level 2 that maximizes $E[t \mid \hat{s}] v(e)-c(e)$.

Finally, we want to compare the selection properties of the different contractual forms with respect to unobserved worker heterogeneity. Under separate contracts, 
according to the prior distribution, the probability of filling the level-2 job with a high-ability worker is $1 / 2$. In case of a combined contract, we have again to distinguish between four possible matches in period 1 . With probability $1 / 4$ we have two high-ability workers matched in period 1, with the same probability two low-ability workers meet in the tournament. With the remaining probability $1 / 2$ there is a heterogeneous match between a low-ability and a high-ability worker on level 1. In that case, under a combined contract the probability of promoting the more talented worker is $\hat{p}\left(\hat{e}_{h}^{c}, \hat{e}_{h}^{c} ; t_{1}\right)>1 / 2$ due to $\hat{p}\left(\hat{e}_{i}, \hat{e}_{j} ; t_{1}\right)=1-\hat{p}\left(\hat{e}_{j}, \hat{e}_{i} ; t_{0}\right)$ and (28). Altogether, the selection quality of an internal labor market with a job-promotion scheme (i.e., of a combined contract) is strictly better than that of external recruiting (i.e., of two separate contracts).

Furthermore, it is interesting to contrast our combined contract with a standard job-promotion tournament where wages are attached to jobs, i.e., tournament prizes are fixed rather than determined by the incentive scheme for the next level. Note that, in our model, the separate contract for hierarchy level 1 corresponds to a standard promotion scheme: The relative performance pay $w_{H}$ can also be interpreted as a fixed wage attached to the next hierarchy level. Since level-1 effort is higher under the combined contract, $\hat{e}_{h}^{c}>\hat{e}_{h}^{s}$, we obtain the following result.

Corollary 1 Combining job-promotion with incentive pay on the next hierarchy level always improves the selection quality of a job-promotion tournament.

Proof. $\hat{p}\left(\hat{e}_{h}^{c}, \hat{e}_{h}^{c} ; t_{1}\right)>\hat{p}\left(\hat{e}_{h}^{s}, \hat{e}_{h}^{s} ; t_{1}\right)$ since $\frac{\partial}{\partial \hat{e}} \hat{p}\left(\hat{e}, \hat{e} ; t_{1}\right)=\hat{p}_{1}\left(\hat{e}, \hat{e} ; t_{1}\right)+\hat{p}_{2}\left(\hat{e}, \hat{e} ; t_{1}\right)$ $\stackrel{(27)}{=} \hat{p}_{1}\left(\hat{e}, \hat{e} ; t_{1}\right)-\hat{p}_{1}\left(\hat{e}, \hat{e} ; t_{0}\right) \stackrel{(29)}{>} 0$.

In the introduction and at the end of Section 3, we mentioned empirical puzzles that contradict standard tournament theory but can be explained in our model. One of these puzzles was that wages are not attached to jobs and, therefore, to hierarchy levels. As has been shown in this section, the selection quality of standard job-promotion tournaments can be significantly improved by replacing wages that are attached to jobs with incentive pay such as a bonus scheme. Hence, missing wages-attached-to-jobs in the empirical literature on firms' wage policies can be nicely explained by the existence of unobserved worker heterogeneity.

To sum up, we have shown that the dominance of an internal labor market over spot contracting and external recruiting continues to hold under unobserved worker heterogeneity. Moreover, with unobserved heterogeneity, we have a double advantage of internal labor markets - better incentives by utilizing workers' rents 
from higher tiers and a better selection quality. If effort and ability are complements, both advantages will reinforce each other. Finally, we have compared an internal labor market structure that combines a job-promotion scheme and incentive pay on higher hierarchy levels with a standard job-promotion rule, which attaches wages to jobs. The result of Corollary 1 has pointed out that the former personnel policy increases the chances of assigning the worker with the higher unobservable talent to the management job.

\subsection{Observed Worker Heterogeneity}

In this section, we extend the basic model to analyze how observed heterogeneity of workers affects optimal employment contracting. In contrast to Section 4.1, where unobserved worker heterogeneity persists across hierarchy levels, we now assume that all workers share the same abilities in the production task, but differ in their managerial talents. On the first hierarchy level, we maintain the assumptions of the basic model. On level 2, however, the firm's valuation of the manager's effort now depends on the latter's ability. In particular, if the firm assigns worker $i$ to the management task, $i$ 's effort choice $e$ yields the value $v(e)+\mu_{i}, i=A, B$. Here, $\mu_{A}$ and $\mu_{B}$ are independent draws from a probability distribution of the same random variable, which reflects workers' different talents for the management position. At the beginning of the first period, nobody knows $\mu_{i}$. However, during the course of that period, the firm gets to know the workers and, finally, can assess who is better suited for the managerial task. Hence, the firm observes $\mu_{i}$ at the end of the first period. No other party is able to assess the workers' suitability for level 2 and, as a consequence, $\mu_{i}$ is non-verifiable. Instead of promoting a worker from level 1 to the management position, the firm can also hire an external candidate of unknown talent. Then, the expected monetary value of managerial effort is $v(e)+E\left[\mu_{i}\right]$.

For simplicity, we assume that ability can be either high or low, $\mu_{i} \in\{0, \mu\}$, where $\mu>0$. The probability that $\mu_{i}$ is high is $1 / 2$. Accordingly, at the end of period 1 , there are four possible situations with respect to the outcome of the tournament:

(i) both winner and loser have high ability,

(ii) only the winner has high ability,

(iii) both have low ability,

(iv) the winner has low ability whereas the loser's ability is high.

In cases (i) and (ii), the firm cannot do better than promoting the tournament 
winner, because he is of the highest possible ability. However, in case (iii), from a pure selection perspective, the firm would be better off by hiring an external candidate for the management position, receiving an expected ability of $\mu / 2$ instead of zero. In case (iv), the firm prefers to promote the loser of the tournament instead of the winner.

Hence, the firm faces the following trade-off: If it ex-ante announces to always promote internally, thereby strengthening level-1 effort incentives, it forgoes the possibility to hire a better suited external candidate for the management job when both workers are of low ability. Moreover, in case (iv), the firm encounters a commitment problem: Since the relative performance signal from level 1 is not verifiable, the firm cannot commit to promote the tournament winner when he is less suited for the management position than his colleague. We assume that the firm can contract to exclusively promote internally, but has no means to commit to promoting the tournament winner in case (iv). ${ }^{25}$

Consequently, the firm can choose between two contractual forms denoted by $C_{1}$ and $C_{2}$. Under contract $C_{1}$, the firm announces to always promote internally. In particular, in cases (i)-(iii), the winner of the tournament becomes the new manager whereas, in case (iv), the tournament loser is promoted. By contrast, under contract $C_{2}$, the firm leaves open the possibility to hire from the external labor market. It announces to promote the winner of the tournament only if he is of high ability, i.e., in cases (i) and (ii). In case (iii), however, the firm recruits the new manager from the external labor market. In case (iv), analogously to $C_{1}$, the tournament loser is promoted.

By choosing between the contracts $C_{1}$ and $C_{2}$, the firm decides on the probability with which the tournament winner is promoted, which we denote by $\alpha_{C}, C=C_{1}, C_{2}$. Furthermore, $\Gamma_{C}$ denotes the expected ability of the worker that is assigned to level 2 under contract $C$. The values of $\alpha_{C}$ and $\Gamma_{C}$ are given in the following table.

\begin{tabular}{|l|l|l|}
\cline { 2 - 3 } \multicolumn{1}{c|}{} & $C_{1}$ & $C_{2}$ \\
\hline$\alpha_{C}$ & $\frac{3}{4}$ & $\frac{1}{2}$ \\
\hline$\Gamma_{C}$ & $\frac{3}{4} \mu$ & $\frac{7}{8} \mu$ \\
\hline
\end{tabular}

Accordingly, contract $C_{1}$ uses the level-2 rent more effectively for level-1 incentive provision because the tournament winner is promoted with higher probability. How-

\footnotetext{
${ }^{25}$ Hence, we do not consider the possibility to engage in relational contracting, where such a promotion rule can be self-enforcing when the firm cares about its future reputation.
} 
ever, contract $C_{2}$ implies a higher expected managerial ability because it allows for external recruitment in case the production workers are of low ability.

Now assume the firm has chosen contract $C$ and consider the workers' decision problems. On level 2, the worker faces the same decision problem as in the basic model. Thus, assume that $e$ satisfies the second-level incentive constraint (1) and, moreover, the second-level participation constraint (2) holds. Then, on the first level, worker $A$ chooses $\hat{e}_{A}$ to maximize

$w_{L}+\hat{p}\left(\hat{e}_{A}, \hat{e}_{B}\right)\left(w_{H}-w_{L}\right)+\left(\alpha_{C} \cdot \hat{p}\left(\hat{e}_{A}, \hat{e}_{B}\right)+\frac{1-\hat{p}\left(\hat{e}_{A}, \hat{e}_{B}\right)}{4}\right)\left[b_{L}+r(e)\right]-\hat{c}\left(\hat{e}_{A}\right)$

with $r(e)$ given by (6), whereas, by choosing $\hat{e}_{B}$, worker $B$ maximizes

$w_{L}+\left(1-\hat{p}\left(\hat{e}_{A}, \hat{e}_{B}\right)\right)\left(w_{H}-w_{L}\right)+\left(\alpha_{C} \cdot\left(1-\hat{p}\left(\hat{e}_{A}, \hat{e}_{B}\right)\right)+\frac{\hat{p}\left(\hat{e}_{A}, \hat{e}_{B}\right)}{4}\right)\left[b_{L}+r(e)\right]-\hat{c}\left(\hat{e}_{B}\right)$

Thus, the symmetric equilibrium on level 1 is implicitly given by

$$
\hat{p}_{1}(\hat{e}, \hat{e})\left(w_{H}-w_{L}+\left(\alpha_{C}-\frac{1}{4}\right)\left[b_{L}+r(e)\right]\right)=\hat{c}^{\prime}(\hat{e}) .
$$

The first-level participation constraint is

$$
w_{L}+\frac{1}{2}\left(w_{H}-w_{L}\right)+\frac{1}{2}\left(\alpha_{C}+\frac{1}{4}\right)\left[b_{L}+r(e)\right]-\hat{c}(\hat{e}) \geq 0 .
$$

Hence, given contract $C_{1}$, the firm has to solve

$$
\begin{gathered}
\max _{\substack{e, \hat{e}, w_{L}, w_{H}, b_{H}, b_{L}}} 2 \hat{v}(\hat{e})-w_{L}-w_{H}+\left[v(e)-b_{L}-p(e)\left(b_{H}-b_{L}\right)\right]+\Gamma_{C_{1}} \\
\text { subject to }(1),(2),(23),(42),(43) .
\end{gathered}
$$

The restrictions (1) and (2) are the incentive and participation constraints, respectively, for level 2. Conditions (42) and (43) are the new incentive constraint and participation constraint, respectively, for level 1. Finally, (23) are the limited-liability constraints. Analogously to the problem of determining the optimal combined contract for homogeneous workers (Proposition 2), we can simplify the firm's problem 
to $^{26}$

$$
\begin{gathered}
\max _{e, \hat{e}} \pi^{C_{1}}(e, \hat{e})+\Gamma_{C_{1}} \quad \text { s.t. } \Delta w(\hat{e})-\frac{1}{2} r(e) \geq 0, \\
\text { where } \pi^{C_{1}}(e, \hat{e})=2 \hat{v}(\hat{e})-\Delta w(\hat{e})+\left[v(e)-c(e)-\frac{1}{2} r(e)\right]
\end{gathered}
$$

Given contract $C_{2}$, the firm solves

$$
\begin{gathered}
\max _{\substack{e, \hat{e}, w_{L}, w_{H}, b_{H}, b_{L}}} 2 \hat{v}(\hat{e})-w_{L}-w_{H}+\frac{3}{4}\left[v(e)-b_{L}-p(e)\left(b_{H}-b_{L}\right)\right]+\frac{1}{4} E+\Gamma_{C_{2}} \\
\text { subject to }(1),(2),(23),(42),(43) .
\end{gathered}
$$

Here, $E$ denotes the firm's level-2 profit when an external candidate is hired for the job on the second hierarchy tier. To such a candidate, the firm offers the optimal separate contract for level 2. Consequently, we have $E=v\left(e^{s}\right)-r\left(e^{s}\right)-c\left(e^{s}\right)$. Proceeding in analogy to contract $C_{1},{ }^{27}$ the firm's problem boils down to

$$
\begin{gathered}
\max _{e, \hat{e}} \pi^{C_{2}}(e, \hat{e})+\Gamma_{C_{2}} \quad \text { s.t. } \Delta w(\hat{e})-\frac{1}{4} r(e) \geq 0, \\
\text { where } \pi^{C_{2}}(e, \hat{e})=2 \hat{v}(\hat{e})-\Delta w(\hat{e})+\frac{3}{4}\left[v(e)-c(e)-\frac{2}{3} r(e)\right]+\frac{1}{4} E
\end{gathered}
$$

Let $\left(\hat{e}_{C}, e_{C}\right)$ denote the effort levels that the firm induces under contract $C$. Then, the firm allows for external recruitment if and only if

$$
\pi^{C_{1}}\left(\hat{e}_{C_{1}}, e_{C_{1}}\right)-\pi^{C_{2}}\left(\hat{e}_{C_{2}}, e_{C_{2}}\right) \leq \Gamma_{C_{2}}-\Gamma_{C_{1}} .
$$

The left-hand side of inequality (45) corresponds to the incentive effect of establishing an exclusively internal labor market (contract $C_{1}$ ) instead of sometimes recruiting on the external labor market (contract $C_{2}$ ). By contrast, the right-hand side of (45) characterizes the beneficial selection effect of external recruitment. Since the left-hand side is independent of $\mu$ while the right-hand side is increasing in $\mu$, we obtain the following result.

Proposition 5 The firm recruits externally with positive probability if workers dif-

\footnotetext{
${ }^{26}$ See the additional pages for the referees.

${ }^{27}$ Under contract $C_{2}$, for a given effort pair $(\hat{e}, e)$, the firm minimizes implementation costs $w_{L}+w_{H}+\frac{3}{4}\left[b_{L}+p(e)\left(b_{H}-b_{L}\right)\right]$.
} 
fer sufficiently strongly in their abilities, i.e., if $\mu$ is sufficiently large.

According to Proposition 5, when the firm is able to observe some worker characteristics that affect productivity on the second hierarchy level, the firm may want to leave open the possibility of external recruitment for the level-2 job. External recruitment occurs with positive probability whenever the firm will suffer a sufficiently large productivity loss if it assigns a low-ability type to the management position. Then, in order to improve selection, the firm sacrifices part of the incentive effect of the level-2 rent for level-1 workers.

As mentioned in the introduction, Baker, Gibbs and Holmström (1994a, 1994b), among others ${ }^{28}$ document external recruiting on higher hierarchy levels. This observation contradicts the strict version of an internal labor market as well as traditional models on job-promotion tournaments since external hiring destroys internal career incentives. According to Proposition 5, the observation of entry ports on higher levels is in line with our model. Such a weaker form of an internal labor market will be favored by the firm if it learns workers' abilities during their careers and selection of appropriate employees for management positions is an important issue relative to incentive provision.

\section{Conclusion}

We analyzed a two-tier hierarchy where workers compete in a rank-order tournament on level 1. On the second tier, a worker carries out a managerial task leading to an individual performance signal, which can be used in a bonus contract. Workers are protected by limited liability on either hierarchy level. We have shown that interlinking the incentive schemes from the two tiers by a job-promotion tournament, thereby creating an internal labor market, has two advantages: First, rents from level 2 can be used to create incentives for level 1. As a consequence, the firm may even implement first-best effort on the second hierarchy level although the worker earns a strictly positive rent on this level. Second, in case of unobserved heterogeneity, a combined contract has the additional advantage of improving the tournament's selection quality in promoting the most talented internal worker. If, however, some characteristics of a worker's ability are observable, the firm may prefer to hire level-2 workers from the external labor market when it turns out that the

\footnotetext{
${ }^{28}$ See footnote 3 .
} 
internal candidates are of low ability. Thus, if selection of high-ability workers is sufficiently important, it may be optimal to have ports of entry on higher hierarchy levels. In this case, level-2 workers hired from the outside market obtain a different contract than internally promoted workers. In particular, external candidates receive lower bonuses and, consequently, lower rents than internal ones. Hence, our analysis implies that firms that utizilize internal labor markets to exploit higherlevel rents for incentive provision on lower tiers should offer lower-powered incentive contracts to external hires than to internal ones.

Combining a promotion tournament with a bonus scheme on the next tier of the hierarchy might lead to further advantages if there is the possibility of sabotage among heterogeneous workers. For example, Münster (2007) shows that more able workers may be deterred from participating in a tournament if contestants can sabotage each other. Then, the advantage of higher talent is completely erased since more able workers are sabotaged more heavily than less able ones, thereby equalizing the winning probabilities of the heterogeneous workers. However, if the winner prize of the tournament is a bonus contract that entails higher rents for more able workers, the problem of adverse participation may be mitigated.

In a different setting, the combination of a tournament with a bonus scheme may be useful to make the competition between heterogeneous contestants more even. As is known from the tournament literature, the more uneven competition the less effort will be chosen in equilibrium. Suppose that unobserved talent and effort are substitutes on each hierarchy level and not complements as in our paper. Then, workers' rents from a bonus contract on the second hierarchy level may be decreasing in ability. As a result, introducing a bonus scheme would mitigate the problem of uneven competition on level 1 . The reason is that more able workers have lower expected rents from winning the tournament than less able ones. If the firm cannot use handicaps (e.g., due to only ordinal information) to counterbalance ability differences, such decreasing rents would be an appropriate instrument for regulating competition. 


\section{Appendix}

\subsection{Proof of Proposition 2}

We can solve problem (21)-(23) in two steps: First, we derive the firm's minimum cost for inducing a given pair of effort levels $(\hat{e}, e)$. Then, we use the optimal cost function to solve the profit maximization problem and determine the optimal effort pair $\left(\hat{e}^{c}, e^{c}\right)$. The cost minimization problem for a given effort pair $(\hat{e}, e)$ reads as

$$
\begin{aligned}
& \min _{w_{L}, w_{H}, b_{L}, b_{H}} 2 w_{L}+\left(w_{H}-w_{L}\right)+b_{L}+p(e)\left(b_{H}-b_{L}\right) \\
& \text { subject to (1), (2), (19), (20), } w_{L}, w_{H}, b_{L}, b_{H} \geq 0 .
\end{aligned}
$$

By the incentive constraint $(1), b_{H}-b_{L}=\frac{c^{\prime}(e)}{p^{\prime}(e)}$. Thus, in combination with the incentive constraint (19), we obtain

$$
w_{H}-w_{L}=\frac{\hat{c}^{\prime}(\hat{e})}{\hat{p}_{1}(\hat{e}, \hat{e})}-b_{L}-p(e) \frac{c^{\prime}(e)}{p^{\prime}(e)}+c(e)=\Delta w(\hat{e})-b_{L}-r(e),
$$

where $\Delta w(\hat{e})$ is given by $(10)$ and $r(e)$ by $(6) .{ }^{29}$

Using (46), the first-level participation constraint (20) boils down to

$$
w_{L}+\frac{1}{2} \Delta w(\hat{e})-\hat{c}(\hat{e}) \geq 0
$$

Furthermore, the second-level participation constraint (2) becomes

$$
b_{L}+p(e) \frac{c^{\prime}(e)}{p^{\prime}(e)}-c(e)=b_{L}+r(e) \geq 0
$$

Thus, substituting for the tournament prize spread $w_{H}-w_{L}$ and the bonus spread $b_{H}-b_{L}$, the cost minimization problem can be simplified to ${ }^{30}$

$$
\begin{gathered}
\min _{w_{L}, b_{L}} 2 w_{L}+\Delta w(\hat{e})+c(e) \quad \text { subject to }(47),(48) \text { and } \\
\Delta w(\hat{e})-b_{L}-r(e)+w_{L}, w_{L}, \quad b_{L} \geq 0 .
\end{gathered}
$$

By Lemma 1, we obtain $b_{L}^{c}=0$ for the optimal low bonus: This satisfies the

\footnotetext{
${ }^{29}$ Recall that $\Delta w(\hat{e})$ is the prize spread necessary to induce $\hat{e}$ under separate contracts. However, note that $\Delta w(\hat{e})$ will usually be different from $w_{H}^{c}-w_{L}^{c}$.

${ }^{30}$ Note that the optimal high bonus, $b_{H}=\frac{c^{\prime}(e)}{p^{\prime}(e)}+b_{L}$, is non-negative due to $b_{L} \geq 0$.
} 
participation constraint for the second hierarchy level (48) and is also best for ensuring that $w_{H}=\Delta w(\hat{e})-b_{L}-r(e)+w_{L} \geq 0$. Hence, we can skip constraint (48) and obtain

$$
\begin{gathered}
\min _{w_{L}} 2 w_{L}+\Delta w(\hat{e})+c(e) \quad \text { subject to }(47) \text { and } \\
\Delta w(\hat{e})-r(e)+w_{L}, w_{L} \geq 0 .
\end{gathered}
$$

The cost-minimizing $w_{L}$ is given by

$$
w_{L}=\max \left\{0, \hat{c}(\hat{e})-\frac{1}{2} \Delta w(\hat{e}), r(e)-\Delta w(\hat{e})\right\} \text {. }
$$

From (13), we know that $\frac{1}{2} \Delta w(\hat{e})-\hat{c}(\hat{e}) \geq 0$. Therefore,

$$
w_{L}=\max \{0, r(e)-\Delta w(\hat{e})\}
$$

We now have to distinguish two cases. The first case is

$$
w_{H}-w_{L}=\Delta w(\hat{e})-r(e) \geq 0 \text {. }
$$

Then, $w_{L}=0$ and $w_{H}=\Delta w(\hat{e})-r(e)$. In the second case,

$$
w_{H}-w_{L}=\Delta w(\hat{e})-r(e)<0 \text {. }
$$

Hence, $w_{L}=r(e)-\Delta w(\hat{e})$ and $w_{H}=0$. In the first case, the firm's expected labor costs are

$$
2 w_{L}+\Delta w(\hat{e})+c(e)=\Delta w(\hat{e})+c(e)
$$

and in the second scenario the firm's costs amount to

$$
2 w_{L}+\Delta w(\hat{e})+c(e)=2 r(e)-\Delta w(\hat{e})+c(e) .
$$

We can now turn to the second step of the solution procedure, the solution of the firm's profit maximization problem. The optimal effort pair $\left(\hat{e}^{c}, e^{c}\right)$ solves

$$
\max _{e, \hat{e}}\left\{\begin{array}{lr}
2 \hat{v}(\hat{e})+v(e)-\Delta w(\hat{e})-c(e) & \text { if } \quad \Delta w(\hat{e})-r(e) \geq 0 \\
2 \hat{v}(\hat{e})+v(e)-[2 r(e)-\Delta w(\hat{e})+c(e)] & \text { otherwise. }
\end{array}\right.
$$


We can see that in case 2 (i.e., the second line of the maximization problem) the firm's objective function is monotonically increasing in $\hat{e}$. Hence, for each $e$, the firm chooses the maximum possible $\hat{e}$, which makes the given restriction just binding, i.e., $\Delta w(\hat{e})=r(e)$. This implies that case 2 becomes a special case of case 1 . Thus, the firm never wants to induce effort levels $(\hat{e}, e)$ such that $\Delta w(\hat{e})<r(e)$. Doing so would imply that $0=w_{H}^{c}<w_{L}^{c}$. Intuitively, this means that, by implementing an adverse relative performance scheme, the firm pays for reducing first-level incentives that stem from the second-level rent $r(e)$. Such a contract cannot be optimal. The firm would be better off by setting $0=w_{H}^{c}=w_{L}^{c}$, thereby increasing first-level effort and reducing workers' first-period rents.

Hence, we are always in the first case. Consequently, $w_{L}^{c}=0$ and the results of the proposition follow.

\subsection{Proof of Proposition 3}

(i) Let $\pi^{s}$ and $\pi^{c}$ denote the firm's respective profits under the two contractual forms, i.e.,

$$
\begin{aligned}
& \pi^{s}:=2 \hat{v}\left(\hat{e}^{s}\right)-\Delta w\left(\hat{e}^{s}\right)+v\left(e^{s}\right)-r\left(e^{s}\right)-c\left(e^{s}\right), \\
& \pi^{c}:=2 \hat{v}\left(\hat{e}^{c}\right)-\Delta w\left(\hat{e}^{c}\right)+v\left(e^{c}\right)-c\left(e^{c}\right) .
\end{aligned}
$$

Under a combined contract, the firm can induce the same level-2 effort as under the optimal separate contracts by offering the bonuses $b_{L}^{s}, b_{H}^{s}$. If the corresponding rent $r\left(e^{s}\right)>0$ does not exceed $\Delta w\left(\hat{e}^{s}\right)$, setting $w_{H}=\Delta w\left(\hat{e}^{s}\right)-r\left(e^{s}\right)$ implements $\hat{e}^{s}$ on level 1. Thus, if $r\left(e^{s}\right) \leq w_{H}^{s}$, there is a combined contract that replicates the effort choices under the optimal separate contracts at strictly lower costs. It follows that $\pi^{c}>\pi^{s}$. If $r\left(e^{s}\right)>\Delta w\left(\hat{e}^{s}\right)$, the combined contract that induces $e^{s}$ on level 2 entails $\hat{e}>\hat{e}^{s}$ on level 1. Thus, profit under the combined contract $\left(0,0, b_{L}^{s}, b_{H}^{s}\right)$ is strictly larger than $\pi^{s}$. Hence, under the optimal combined contract, we must also have $\pi^{c}>\pi^{s}$.

(ii) $\hat{e}^{c}=\hat{e}^{s}$ immediately follows from examining the objective functions (14) and (24). $e^{c}>e^{s}$ follows from $r^{\prime}(e)>0$, which we have proven in Lemma 1 , and $r^{\prime \prime}(e)>0$, which follows from our regularity assumptions and is straightforward to check.

It remains to prove result (iii). Due to the binding restriction, we can consider 
$e$ as an implicitly defined function of $\hat{e}$, i.e., $e(\hat{e})$ with

$$
\frac{\partial e}{\partial \hat{e}}=\frac{\Delta w^{\prime}(\hat{e})}{r^{\prime}(e)}>0
$$

Moreover, the firm's objective function (24) becomes

$$
2 \hat{v}(\hat{e})+v(e(\hat{e}))-\Delta w(\hat{e})-c(e(\hat{e})) .
$$

The respective first-order condition is

$$
2 \hat{v}^{\prime}(\hat{e})-\Delta w^{\prime}(\hat{e})+\left[v^{\prime}(e(\hat{e}))-c^{\prime}(e(\hat{e}))\right] \frac{\partial e}{\partial \hat{e}}=0 .
$$

Hence, compared to the case where the restriction is non-binding, we either have higher effort at hierarchy level 1 and lower effort at level 2, or vice versa. Inserting $\partial e / \partial \hat{e}$ in (50) yields

$$
2 \hat{v}^{\prime}(\hat{e})+\frac{v^{\prime}(e(\hat{e}))-c^{\prime}(e(\hat{e}))-r^{\prime}(e(\hat{e}))}{r^{\prime}(e(\hat{e}))} \Delta w^{\prime}(\hat{e})=0 .
$$

Recall that $\Delta w^{\prime}(\hat{e})>0$ and $r^{\prime}(e)>0$. The optimal effort, $e^{c}$, must therefore satisfy $v^{\prime}\left(e^{c}\right)-c^{\prime}\left(e^{c}\right)-r^{\prime}\left(e^{c}\right)<0$. Under separate contracts, we have $v^{\prime}\left(e^{s}\right)-c^{\prime}\left(e^{s}\right)-r^{\prime}\left(e^{s}\right)=$ 0 . Thus, since $v(e)-c(e)-r(e)$ is strictly concave, it follows that $e^{c}>e^{s}$.

Now consider the effort choice on hierarchy level 1 under a binding restriction (25). Suppose that the firm wants to implement the same effort level as under a non-binding restriction, i.e., $\hat{e}^{s}=\arg \max _{\hat{e}}\{2 \hat{v}(\hat{e})-\Delta w(\hat{e})\}$. However, since (25) is binding in this situation, the corresponding level-2 effort is below the optimal one, $e^{F B}$. Of course, the firm can raise $e$ to increase $v(e)-c(e)$, but then it has to increase $\hat{e}$ as well because of $\partial e / \partial \hat{e}>0$. Whether such an adjustment is beneficial to the firm or not depends on the functional forms. In any case, since both functions $2 \hat{v}(\hat{e})-\Delta w(\hat{e})$ and $v(e)-c(e)$ are strictly concave, the firm will never raise $e$ above $e^{F B}$. This is because, if $e>e^{F B}$ and $\hat{e}>\hat{e}^{s}$, the firm can increase profits by decreasing both effort levels, while keeping (25) binding. This proves $e^{c}<e^{F B}$.

Since $e^{c}<e^{F B}$ implies $v^{\prime}\left(e^{c}\right)-c^{\prime}\left(e^{c}\right)>0$, from (50) we obtain that the corresponding optimal effort on hierarchy level 1 must satisfy $2 \hat{v}^{\prime}(\hat{e})-\Delta w^{\prime}(\hat{e})<0$. Thus, this effort must be larger than the optimal level-1 effort under a non-binding restriction (25). Since that effort was identical with the optimal level-1 effort under 
separate contracts, $\hat{e}^{s}$, we have $\hat{e}^{c}>\hat{e}^{s}$ under the binding restriction.

\section{References}

Ariga, K., Ohkusa, Y. and G. Brunello (1999): Fast Track: Is It in the Genes? The Promotion Policy of a Large Japanese Firm. Journal of Economic Behavior and Organization 38, 385-402.

Baker, G.P., Gibbs, M. and B. Holmström (1994a): The Internal Economics of the Firm: Evidence from Personnel Data. Quarterly Journal of Economics 109, 881-919.

Baker, G.P., Gibbs, M. and B. Holmström (1994b): The Wage Policy of a Firm. Quarterly Journal of Economics 109, 921-955.

Demougin, D. and C. Fluet (2001): Monitoring Versus Incentives. European Economic Review 45, 1741-1764.

Demougin, D. and D. Garvie (1991): Contractual Design with Correlated Information under Limited Liability. RAND Journal of Economics 22, 477-487.

Dixit, A. (1987): Strategic Behavior in Contests. American Economic Review 77, 891-898.

Doeringer, P.B. (1986): Internal Labor Markets and Noncompeting Groups, American Economic Review, Papers and Proceedings 76, 48-52.

Doeringer, P.B. and M.J. Piore (1971): Internal Labor Markets and Manpower Analysis. Lexington.

Dohmen, T.J., Kriechel, B. and G.A. Pfann (2004): Monkey Bars and Ladders: The Importance of Lateral and Vertical Job Mobility in Internal Labor Market Careers. Journal of Population Economics 17, 193-228.

Endo, K. (1994): Satei (Personal Assessment) and Interworker Competition in Japanese Firms, Industrial Relations 33, 70-82.

Gibbons, R. and M. Waldman (1999): A Theory of Wage and Promotion Dynamics Inside Firms. Quarterly Journal of Economics 114, 1321-1358. 
Gibbs, M. and W. Hendricks (2004): Do Formal Salary Systems Really Matter?. Industrial and Labor Relations Review 58, 71-93.

Grund, C. (2005): The Wage Policy of Firms - Comparative Evidence for the U.S. and Germany from Personnel Records. International Journal of Human Resource Management 16, 104-119.

Harris, M. and B. Holmström (1982): A Theory of Wage Dynamics. Review of Economic Studies 49, 315-333.

Holmström, B. (1999): Managerial Incentive Problems: A Dynamic Perspective. Review of Economic Studies 66, 169-182.

Ichniowski, C., Shaw, K. and G. Prennushi (1997): The Effects of Human Resource Management Practices on Productivity: A Study of Steel Finishing Lines, American Economic Review 87, 291-313.

Koike, K. (1988): Understanding Industrial Relations in Modern Japan. St. Martin's Press, New York.

Laffont, J.-J. and D. Martimort (2002): The Theory of Incentives. Princeton University Press.

Lazear, E.P. (1992): The Job as a Concept. In: W.J. Bruns, Jr. (Ed.): Performance Measurement, Evaluation, and Incentives. Harvard Business School Press: Boston, 183-215.

Lazear, E.P. and P. Oyer (2004): Internal and External Labor Markets: A Personnel Economics Approach, Labour Economics 11, 527-554.

Lazear, E.P. and S. Rosen (1981): Rank-Order Tournaments as Optimum Labor Contracts. Journal of Political Economy 89, 841-864.

Lindbeck, A. and D.J. Snower (2000): Multitask Learning and the Reorganization of Work: From Tayloristic to Holistic Organization, Journal of Labor Economics 18, 353-376.

Malcomson, J.M. (1984): Work Incentives, Hierarchy, and Internal Labor Markets. Journal of Political Economy 92, 486-507. 
Malcomson, J.M. (1986): Rank-Order Contracts for a Principal with Many Agents. Review of Economic Studies 53, 807-817.

Milgrom, P.R. (1981): Good News and Bad News: Representation Theorems and Applications. Bell Journal of Economics 12, 380-391.

Münster, J. (2007): Selection Tournaments, Sabotage, and Participation. Journal of Economics and Management Strategy 16, 943-970.

Murphy, K.J. (1986): Incentives, Learning, and Compensation: A Theoretical and Empirical Investigation of Managerial Labor Contracts. RAND Journal of Economics 17, 59-76.

Nalebuff, B.J. and J.E. Stiglitz (1983): Prizes and Incentives: Towards a General Theory of Compensation and Competition. Bell Journal of Economics 3, $21-43$.

Ohlendorf, S. and P.W. Schmitz (2008): Repeated Moral Hazard, Limited Liability, and Renegotiation. CEPR Discussion paper No. 6725.

Schmitz, P.W. (2005): Workplace Surveillance, Privacy Protection, and Efficiency Wages, Labour Economics 12, 727-738.

Schöttner, A. and V. Thiele (forthcoming): Promotion Tournaments and Individual Performance Pay, Journal of Economics and Management Strategy.

Seltzer, A. and D. Merrett (2000): Personnel Practices at the Union Bank of Australia: Panel Evidence from the 1887-1900 Entry Cohorts. Journal of Labor Economics 18, 573-613.

Tirole, J. (1999): Incomplete Contracts: Where Do We Stand?, Econometrica 67, $741-781$.

Treble, J., van Gameren, E., Bridges, S. and T. Barmby (2001): The Internal Economics of the Firm: Further Evidence from Personnel Data. Labour Economics 8, 531-552.

Tsoulouhas, T., Knoeber, C. and A. Agrawal (2007): Contests to Become CEO: Incentives, Selection and Handicaps. Economic Theory 30, 195-221. 
Waldman, M. (forthcoming): Theory and Evidence in Internal Labor Markets. Handbook of Organizational Economics.

Williamson, O.E., Wachter, M.L. and J.E. Harris (1975): Understanding the Employment Relation: The Analysis of Idiosyncratic Exchange, Bell Journal of Economics 6, 250-278. 


\section{Appendix for Referees}

\subsection{Modified Limited-Liability Constraints}

In this section, we reconsider the problem (21)-(22) of a combined contract in the basic model where the limited-liability constraints (23) are replaced by $w_{L} \geq 0$, $w_{H}+b_{L} \geq 0$ and $w_{H}+b_{H} \geq 0$. We will show that these modifications do not change our results. Again, we start with minimizing the firm's cost for inducing a given pair of effort levels $(\hat{e}, e)$ :

$$
\min _{w_{L}, w_{H}, b_{L}, b_{H}} 2 w_{L}+\left(w_{H}-w_{L}\right)+b_{L}+p(e)\left(b_{H}-b_{L}\right)
$$

subject to (1), (2), (19), (20), $w_{L}, b_{L}+w_{H}, b_{H}+w_{H} \geq 0$.

From the incentive constraint (1) we obtain $b_{H}-b_{L}=\frac{c^{\prime}(e)}{p^{\prime}(e)}$, which, in combination with the incentive constraint (19), yields

$$
w_{H}-w_{L}=\frac{\hat{c}^{\prime}(\hat{e})}{\hat{p}_{1}(\hat{e}, \hat{e})}-b_{L}-p(e) \frac{c^{\prime}(e)}{p^{\prime}(e)}+c(e)=\Delta w(\hat{e})-b_{L}-r(e),
$$

where $\Delta w(\hat{e})$ is given by (10) and $r(e)$ by (6). Using this expression, (20) can be rewritten as $w_{L}+\frac{1}{2} \Delta w(\hat{e})-\hat{c}(\hat{e}) \geq 0$. Furthermore, $(2)$ becomes $b_{L}+r(e) \geq 0$. In addition, we have

$$
\begin{aligned}
& b_{L}+w_{H}=b_{L}+\Delta w(\hat{e})-b_{L}-r(e)+w_{L}=\Delta w(\hat{e})-r(e)+w_{L} \\
& b_{H}+w_{H}=b_{H}+\Delta w(\hat{e})-b_{L}-r(e)+w_{L}=\frac{c^{\prime}(e)}{p^{\prime}(e)}+\Delta w(\hat{e})-r(e)+w_{L} .
\end{aligned}
$$

Substituting for $w_{H}-w_{L}$ and $b_{H}-b_{L}$ in the objective function, the cost minimization problem can be summarized as follows:

$$
\begin{gathered}
\min _{w_{L}, b_{L}} 2 w_{L}+\Delta w(\hat{e})+c(e) \\
\text { subject to } w_{L}+\frac{1}{2} \Delta w(\hat{e})-\hat{c}(\hat{e}) \geq 0 \\
b_{L}+r(e) \geq 0 \\
w_{L}, \Delta w(\hat{e})-r(e)+w_{L}, \frac{c^{\prime}(e)}{p^{\prime}(e)}+\Delta w(\hat{e})-r(e)+w_{L} \geq 0 .
\end{gathered}
$$


The last non-negativity constraint is less strong than the second one and can thus be skipped. Since $b_{L}$ does not appear in the objective function but only in the second-level participation constraint, we can set $b_{L}=0$ (or any other $b_{L} \geq-r(e)$ ). Moreover, since $\frac{1}{2} \Delta w(\hat{e})-\hat{c}(\hat{e}) \geq 0$, the first constraint is satisfied whenever $w_{L} \geq 0$ and can, therefore, also be skipped. Altogether, we obtain the same cost minimization problem as in Subsection 6.1 (Proof of Proposition 2), where we assumed $w_{L}, w_{H}, b_{L}, b_{H} \geq 0$. The intuition is as follows. If $w_{L}, b_{L}+w_{H}, b_{H}+w_{H} \geq 0$, a negative bonus $b_{L}$ can be used to decrease rents on the second tier. However, all these rents serve as indirect incentives for the first tier. Hence, these rents do not constitute costs for the firm so that it cannot benefit from lowering them. Finally, if the rents are so high that they provide too strong incentives for the first tier, $w_{H}=0$ anyway.

\subsection{Combined Contract under Unobserved Heterogeneity}

Step 1: Minimizing costs

Since $b_{H} \geq 0$ is ensured by the incentive constraint for hierarchy level 2 in combination with $b_{L} \geq 0$ the problem of minimizing implementation costs reduces to

$$
\begin{gathered}
\min _{w_{L}, w_{H}, b_{L}} \Delta \tilde{w}(\hat{e})+2 w_{L}+c(e) \\
\text { subject to } b_{L}+r(e) \geq 0 \\
w_{L}+\frac{1}{2} \Delta \tilde{w}(\hat{e})-\hat{c}(\hat{e}) \geq 0 \\
w_{H}-w_{L}+b_{L}+r(e)=\Delta \tilde{w}(\hat{e}) \\
w_{H}, w_{L}, b_{L} \geq 0 .
\end{gathered}
$$

Replacing $w_{H}$ yields:

$$
\begin{gathered}
\min _{w_{L}, b_{L}} \Delta \tilde{w}(\hat{e})+2 w_{L}+c(e) \\
\text { subject to } b_{L}+r(e) \geq 0 \\
w_{L}+\frac{1}{2} \Delta \tilde{w}(\hat{e})-\hat{c}(\hat{e}) \geq 0 \\
\Delta \tilde{w}(\hat{e})-b_{L}-r(e)+w_{L}, w_{L}, b_{L} \geq 0 .
\end{gathered}
$$

From Lemma 1 we know that $r(e) \geq 0$ so that $b_{L}^{c}=0$ and the minimization problem 
further reduces to

$$
\begin{gathered}
\min _{w_{L}} \Delta \tilde{w}(\hat{e})+2 w_{L}+c(e) \\
\text { s.t. } w_{L}+\frac{1}{2} \Delta \tilde{w}(\hat{e})-\hat{c}(\hat{e}) \geq 0 \\
\Delta \tilde{w}(\hat{e})-r(e)+w_{L}, w_{L} \geq 0 .
\end{gathered}
$$

Hence,

$$
w_{L}=\max \left\{0, \hat{c}(\hat{e})-\frac{1}{2} \Delta \tilde{w}(\hat{e}), r(e)-\Delta \tilde{w}(\hat{e})\right\} .
$$

We know that $\frac{1}{2} \Delta \tilde{w}(\hat{e})-\hat{c}(\hat{e}) \geq 0$; otherwise, $\hat{e}$ would not be an equilibrium strategy. Thus,

$$
w_{L}=\max \{0, r(e)-\Delta \tilde{w}(\hat{e})\} .
$$

We have to distinguish two cases. First, $w_{H}-w_{L}=\Delta \tilde{w}(\hat{e})-r(e) \geq 0$. Then,

$$
w_{L}=0 \quad \text { and } \quad w_{H}=\Delta \tilde{w}(\hat{e})-r(e) .
$$

Second, $w_{H}-w_{L}=\Delta \tilde{w}(\hat{e})-r(e)<0$. Then,

$$
w_{L}=r(e)-\Delta \tilde{w}(\hat{e}) \quad \text { and } \quad w_{H}=0 .
$$

In the first case, the firm's expected labor costs are

$$
\Delta \tilde{w}(\hat{e})+2 w_{L}+c(e)=\Delta \tilde{w}(\hat{e})+c(e)
$$

and in the second they amount to

$$
\Delta \tilde{w}(\hat{e})+2 w_{L}+c(e)=2 r(e)-\Delta \tilde{w}(\hat{e})+c(e) .
$$

Step 2: Maximizing expected profits

Therefore, the optimal effort pair $\left(\hat{e}^{c}, e^{c}\right)$ solves

$$
\max _{\hat{e}, e}\left\{\begin{array}{l}
2 E[t] \hat{v}(\hat{e})+E[t \mid \hat{s}] v(e)-\Delta \tilde{w}(\hat{e})-c(e) \text { if } \Delta \tilde{w}(\hat{e})-r(e) \geq 0 \\
2 E[t] \hat{v}(\hat{e})+E[t \mid \hat{s}] v(e)-2 r(e)+\Delta \tilde{w}(\hat{e})-c(e) \quad \text { otherwise. }
\end{array}\right.
$$

In analogy to the basic model, again the firm's objective function in the second line is monotonically increasing in $\hat{e}$ (recall that $\partial E[t \mid \hat{s}] / \partial \hat{e}>0$ according to (38)). 
Hence, for each $e$ the firm chooses the maximum possible $\hat{e}$ that makes the given restriction just bind so that the second line becomes a special case of the problem in line 1. The firm chooses $w_{L}^{c}=0$ and implements the effort pair $\left(\hat{e}_{h}^{c}, e_{h}^{c}\right)$ with

$$
\begin{gathered}
\left(\hat{e}_{h}^{c}, e_{h}^{c}\right) \in \arg \max _{\hat{e}, e}\{2 E[t] \hat{v}(\hat{e})+E[t \mid \hat{s}] v(e)-\Delta \tilde{w}(\hat{e})-c(e)\} \\
\text { subject to } \Delta \tilde{w}(\hat{e})-r(e) \geq 0 .
\end{gathered}
$$

\subsection{Simplification of the Firm's Problem under Contract $A$}

The solution procedure is analogous to the one in Proposition 2. First, we consider the firm's problem of minimizing implementation costs for a given pair of effort levels $(\hat{e}, e)$.

$$
\min _{w_{L}, w_{H}, b_{H}, b_{L}} w_{L}+w_{H}+b_{L}+p(e)\left(b_{H}-b_{L}\right) \quad \text { s.t. (1), (2), (23), (42), (43). }
$$

By using (10), the incentive constraint (42) can be rewritten as

$$
w_{H}-w_{L}=\Delta w(\hat{e})-\left(\alpha_{A}-\frac{1}{4}\right)\left[b_{L}+r(e)\right]
$$

so that the first-level participation constraint (43) boils down to

$$
w_{L}+\frac{1}{2} \Delta w(\hat{e})+\frac{1}{4}\left[b_{L}+r(e)\right]-\hat{c}(\hat{e}) \geq 0 .
$$

Recall that the second-level participation constraint can be written as

$$
b_{L}+r(e) \geq 0
$$

Using (51) together with $\alpha_{A}=\frac{3}{4}$ and $p(e)\left(b_{H}-b_{L}\right)=r(e)+c(e)$, the cost minimization problem reads as ${ }^{31}$

$$
\begin{gathered}
\min _{w_{L}, b_{L}} 2 w_{L}+\Delta w(\hat{e})+c(e)+\frac{1}{2}\left[b_{L}+r(e)\right] \quad \text { s.t. }(52),(53) \text { and } \\
\Delta w(\hat{e})-\frac{1}{2}\left[b_{L}+r(e)\right]+w_{L}, w_{L}, b_{L} \geq 0 .
\end{gathered}
$$

\footnotetext{
${ }^{31}$ Note that $b_{H} \geq 0$ is guaranteed.
} 
Again, $b_{L}=0$ is optimal. Hence, we get rid of constraint (53) and obtain

$$
\min _{w_{L}} 2 w_{L}+\Delta w(\hat{e})+c(e)+\frac{1}{2} r(e) \text { s.t. }(52) \text { and } \Delta w(\hat{e})-\frac{1}{2} r(e)+w_{L}, w_{L} \geq 0 .
$$

The cost-minimizing $w_{L}$ is thus given by

$$
w_{L}=\max \left\{0, \hat{c}(\hat{e})-\frac{1}{2} \Delta w(\hat{e})-\frac{1}{4} r(e), \frac{1}{2} r(e)-\Delta w(\hat{e})\right\} .
$$

From (13), we know that $\frac{1}{2} \Delta w(\hat{e})-\hat{c}(\hat{e}) \geq 0$. Hence, $w_{L}=\max \left\{0, \frac{1}{2} r(e)-\Delta w(\hat{e})\right\}$. We now have to distinguish two cases. The first case is $w_{H}-w_{L}=\Delta w(\hat{e})-\frac{1}{2} r(e) \geq$ 0 . Then, $w_{L}=0$ and $w_{H}=\Delta w(\hat{e})-\frac{1}{2} r(e)$. In the second case, $w_{H}-w_{L}=$ $\Delta w(\hat{e})-\frac{1}{2} r(e)<0$. Hence, $w_{L}=\frac{1}{2} r(e)-\Delta w(\hat{e})$ and $w_{H}=0$. In the first case, the firm's expected labor costs are $2 w_{L}+\Delta w(\hat{e})+c(e)+\frac{1}{2} r(e)=\Delta w(\hat{e})+c(e)+\frac{1}{2} r(e)$, and in the second scenario the firm's costs amount to $2 w_{L}+\Delta w(\hat{e})+c(e)+\frac{1}{2} r(e)=$ $\frac{3}{2} r(e)-\Delta w(\hat{e})+c(e)$. We can now turn to the final step of the solution procedure, the solution of the firm's profit maximization problem. The optimal effort combination solves

$$
\max _{e, \hat{e}} \begin{cases}2 \hat{v}(\hat{e})+v(e)-\Delta w(\hat{e})-c(e)-\frac{1}{2} r(e)+\Gamma_{A} & \text { if } \Delta w(\hat{e})-\frac{1}{2} r(e) \geq 0 \\ 2 \hat{v}(\hat{e})+v(e)-\left[\frac{3}{2} r(e)-\Delta w(\hat{e})+c(e)\right]+\Gamma_{A} & \text { otherwise. }\end{cases}
$$

With the same argumentation as in the proof of Proposition 2, it follows that the firm will never implement effort levels $(\hat{e}, e)$ such that $\Delta w(\hat{e})-\frac{1}{2} r(e)<0$. Thus, $w_{L}=0$ and the firm's optimization problem is given by (44). 